\title{
STABILIZATION OF GRAVITY WATER WAVES
}

\author{
THOMAS ALAZARD \\ CNRS \& ÉCOLE NORMALE SUPÉRIEURE
}

\begin{abstract}
This paper is devoted to the stabilization of the incompressible Euler equation with free surface. We study the damping of two-dimensional gravity waves by an absorbing beach where the water-wave energy is dissipated by using the variations of the external pressure.
\end{abstract}

\section{INTRODUCTION}

Many problems in water-wave theory require to study the behavior of waves propagating in an unbounded domain, like those encountered in the open sea. On the other hand, the numerical analysis of the water-wave equations requires to work in a bounded domain. This problem appears for the effective modeling of many partial differential equations and several methods have been developed to solve it. A classical approach consists in truncating the domain by introducing an artificial boundary. This is possible provided that one can find some special non-reflecting boundary conditions which make the artificial boundary (approximatively) invisible to outgoing waves. We refer to the extensive surveys by Israeli and Orszag [20], Tsynkov [38] and also to the recent papers by Abgrall, Carney, Jennings, Karni, Pridge and Rauch $[22,21]$ for the study of absorbing boundary conditions for the linearized 2D gravity water-wave equations. Another method, which is widespread to study wave equations, consists in damping outgoing waves in an absorbing zone surrounding the computational boundary (see $[20,38,8]$ ). For the water-wave equations, the idea of using the latter method goes back to Le Méhauté [27] in 1972. This approach is very important for the analysis of the water-wave equations for at least two reasons. Firstly, it is used in many numerical studies (we refer to $[11,19,15,17,9,13,18]$ and the references there in) as an efficient approach to absorb outgoing waves. Secondly, the idea of adding an absorbing layer is also useful for the experimental study of water waves in wave basins. Indeed, think of a rectangular wave basin, having vertical walls, equipped with a wave-maker at one extremity. The waves generated by the wave-maker will be reflected at the opposite side and then will interact with the wave produced by the wave-maker. Consequently, to simulate experimentally the open sea propagation, one has to introduce wave absorbers to minimize wave reflection.

The mathematical study of the damping properties of these absorbers corresponds to the mathematical question of the stabilization of the water-wave equations. Our goal in this paper is to start the analysis of this problem for the nonlinear water-wave equations.

This work is partly supported by the grant "ANAÉ" ANR-13-BS01-0010-03. 
There is a huge literature about the absorption of water-wave energy. We refer the reader to the literature review by Ouellet and Datta [33] for a description of the energy absorbing devices commonly used in 48 wave basins around the world. The most popular wave absorbers are passive absorbers. They consist of a beach with a mild slope. The principle is that, when arriving to the artificial beach, the steepening of the forward face of waves and their subsequent overturning dissipates energy. Another widely used strategy is to introduce a porous media to absorb the wave energy. The mathematical analysis of these absorbing devices raises extremely difficult questions. Consequently, to stabilize the water-wave equations or to develop numerical absorbing sponges, one prefers to use simpler means to dissipate energy. For similar problems, the simplest choice could be to use viscous damping, but this is not possible here since one considers a potential flow (so that the velocity is harmonic). For such a flow, the energy can only be transmitted or dissipated through the free surface. This suggests to consider a pneumatic wave maker, that is to say a wave maker where the variations of the external pressure acting on the free surface are used to absorb waves. This idea goes back to the work by Larsen and Dancy [26]. It has been widely used and many elaborations and variants have been implemented, in particular by Clément [14] who proposed to couple the pneumatic wave-maker with a piston-like absorbing boundary condition at the tank extremity (see also [11, 19, 15, 17, 9, 13, 18]).

Let us be more specific. Denote by $\mathcal{H}$ the energy of the fluid and by $P_{\text {ext }}$ the evaluation of the external pressure at the free surface. The question is to find an expression of $P_{\text {ext }}$ in terms of the unknowns such that the following two properties hold:

(1) $P_{\text {ext }}$ vanishes away from the artificial beach (also called sponge layer) which is the neighborhood of the boundary where one wants to absorb the waves;

(2) the energy $\mathcal{H}$ goes to zero (one also wants to determine the rate of decay).

One can easily compute the work done by $P_{\text {ext }}$ (see $\S 2.3$ ) and obtain that

$$
\frac{d \mathcal{H}}{d t}=-\int_{S_{\text {beach }}} P_{\text {ext }} \phi_{n} \mathrm{~d} \sigma
$$

where $S_{\text {beach }}$ is the absorbing zone and $\phi_{n}$ denotes the normal derivative of the velocity potential $\phi$. As noted by Cao, Beck and Schultz ([11]), this suggests to set

$$
P_{\text {ext }}=\chi \phi_{n},
$$

where $\chi \geq 0$ is a cut-off function. Indeed, with this choice it is obvious that the energy is a non-increasing function. The previous observation explains why this choice is widespread (see $[19,9,18]$ and the references there in).

However, to study the stabilization of the water-wave equations, the idea of choosing (1) is inapplicable for the simple reason that the Cauchy problem seems ill-posed when $P_{\text {ext }}$ is given by (1). This question will be studied in a separate paper. Let us only mention that it is a non trivial problem. Indeed, one can modify slightly (1) and obtain a system of equations whose Cauchy problem is well-posed. Namely, if one replaces the normal derivative $\phi_{n}$ by the derivative of $\phi$ in the vertical direction, then the Cauchy problem is well-posed. However, one cannot use the latter choice to stabilize the equations since one cannot prove that the energy is decaying. 
Many other choices for $P_{\text {ext }}$ have been used (see for instance the papers by Baker, Meiron and Orszag [5] and Clamond et al. [13]) but we have not been able to use one of them for the same reasons (either the Cauchy problem is not well-posed or one cannot prove that the energy is decaying). To overcome this problem, we shall take benefit of an elementary (though seemingly new) observation which shows that the energy is decaying when $P_{\text {ext }}$ satisfies

$$
\partial_{x} P_{e x t}=\chi(x) \int_{-h}^{\eta(t, x)} \phi_{x}(t, x, y) \mathrm{d} y,
$$

where $\chi \geq 0$ is a cut-off function, $x$ (resp. $y$ ) is the horizontal (resp. vertical) space variable and $\eta$ is the free surface elevation. By contrast with (1), one can easily prove that the Cauchy problem is well-posed when $P_{\text {ext }}$ satisfies (2). In addition, by exploiting several hidden cancellations, we will be able to quantity the decay rate, that is to estimate the ratio $\mathcal{H}(T) / \mathcal{H}(0)$. By assuming that the solution exists on large time interval, this will imply that the energy converges exponentially to zero.

To conclude this introduction, let us mention that we study only the stabilization problem in this paper and we refer to $[3,23,34,35,36]$ for the analysis of the generation of water waves in a pneumatic wave maker.

Organization of the paper. We gather the statements of our main results in Section 2. Our first main result is an integral identity (see Theorem 2.1) which allows to compare the integral in time of the energy to the work done by the external pressure. This identity, which holds for any solution and any external pressure, will be proved in Section 5 by adapting the multiplier method to the water-wave problem. Since we do not assume that the reader is familiar with control theory, before proving this result we will recall in Section 4 some important methods and results. We will also explain the main difficulties one has to cope with when adapting these methods to the study of the water-wave equations.

As already mentioned, the energy decays when $P_{\text {ext }}$ is given either by (1) or (2). In addition, as we will see in Section 3, the Cauchy problem is well-posed when $P_{\text {ext }}$ is given by (2). This is why we assume that $P_{\text {ext }}$ is given by (2). Our second main result, which is Theorem 2.3, asserts that, by exploiting the integral identity alluded to above, one can quantify the decay rate of the energy for small enough solutions. Assuming that the solution exists on large time intervals, we will obtain an exponential decay (cf Corollary 2.5). This result is stated in Section 2 and proved in Section 6. The latter result holds under a natural assumption about the frequency localization of the solution.

Eventually, in Appendix A we will prove Sobolev estimates for the linearized problem. Also, in Appendix $\mathrm{C}$ we will prove another integral identity, which is not used to prove a stabilization result, but gives an interesting observability inequality.

Acknowledgements. I gratefully acknowledge Félicien Bonnefoy and Guillaume Ducrozet for a demonstration of the wave tank of the École Centrale de Nantes. I would like also to warmly thank Nicolas Burq, Jean-Michel Coron, Emmanuel Dormy and Camille Laurent for stimulating discussions. 


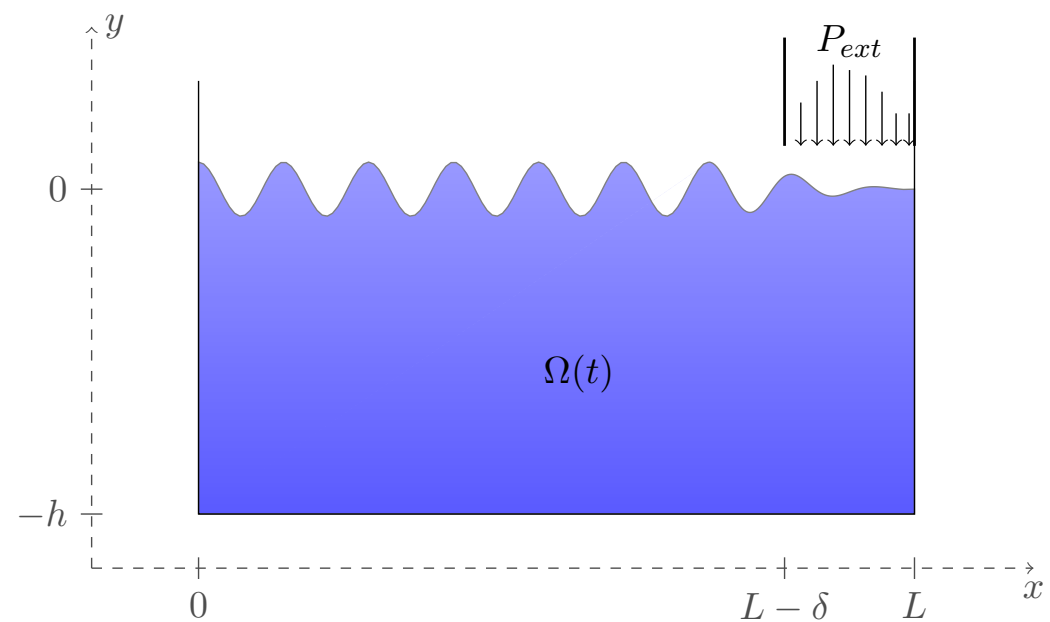

FigURE 1 . Waves generated near $x=0$, propagating to the right, and absorbed in the neighborhood of $x=L$ by means of an external counteracting pressure produced by blowing above the free surface.

\section{MAIN RESUlts}

2.1. The equations. We assume that the dynamics is described by the incompressible Euler equations with free surface and consider the irrotational case. For the sake of simplicity, we consider a two-dimensional fluid located inside a rectangular tank. The water depth is denoted by $h$, the length by $L$ and the free surface elevation by $\eta$. At time $t$, the fluid domain is thus given by

$$
\Omega(t)=\{(x, y): x \in[0, L],-h \leq y \leq \eta(t, x)\},
$$

where $x$ (resp. $y$ ) is the horizontal (resp. vertical) space variable.

Then the velocity is given by $v=\nabla_{x, y} \phi$ for some potential $\phi: \Omega \rightarrow \mathbb{R}$ satisfying

$$
\Delta_{x, y} \phi=0, \quad \partial_{t} \phi+\frac{1}{2}\left|\nabla_{x, y} \phi\right|^{2}+P+g y=0,
$$

where $P: \Omega \rightarrow \mathbb{R}$ is the pressure, $g$ is the acceleration of gravity, $\nabla_{x, y}=\left(\partial_{x}, \partial_{y}\right)$ and $\Delta_{x, y}=\partial_{x}^{2}+\partial_{y}^{2}$. Partial differentiation will be denoted by suffixes, so that $\phi_{x}=\partial_{x} \phi$ and $\phi_{y}=\partial_{y} \phi$ (except for $\partial_{x} P_{\text {ext }}$ ). Furthermore, the velocity satisfies the solid wall boundary condition on the bottom and the vertical walls, which implies that

$$
\begin{array}{ll}
\phi_{x}=0 & \text { for } \quad x=0 \text { or } x=L, \\
\phi_{y}=0 & \text { for } \quad y=-h .
\end{array}
$$

The problem is then determined by two boundary conditions on the free surface. The first equation asserts that the free surface moves with the fluid:

$$
\partial_{t} \eta=\left.\sqrt{1+\eta_{x}^{2}} \phi_{n}\right|_{y=\eta}=\phi_{y}(t, x, \eta)-\eta_{x}(t, x) \phi_{x}(t, x, \eta) .
$$

The second equation is a balance of forces across the free surface. It reads

$$
\left.P\right|_{y=\eta}=P_{\text {ext }},
$$

where $P_{\text {ext }}=P_{\text {ext }}(t, x)$ is the evaluation of the external pressure at the free surface. 
Also we always assume (without explicitly recalling this condition below) that

$$
\eta \geq-\frac{h}{2}, \quad \int_{0}^{L} \eta(t, x) \mathrm{d} x=0 \text { for all time } t .
$$

One can assume that the mean value of $\eta$ vanishes since it is a conserved quantity. We also assume that the free surface intersects the vertical walls ${ }^{1}$ orthogonally:

$$
\eta_{x}=0 \quad \text { for } \quad x=0 \text { or } x=L .
$$

Following Zakharov [41] and Craig-Sulem [16], we work with the evaluation of $\phi$ at the free boundary

$$
\psi(t, x):=\phi(t, x, \eta(t, x)) .
$$

Notice that $\phi$ is fully determined by its trace $\psi$ since $\phi$ is harmonic and satisfies $\phi_{n}=0$ on the walls and the bottom. Now, to obtain a system of two evolution equations for $\eta$ and $\psi$, one introduces the Dirichlet to Neumann operator $G(\eta)$ that relates $\psi$ to the normal derivative of the potential by

$$
G(\eta) \psi=\left.\sqrt{1+\eta_{x}^{2}} \phi_{n}\right|_{y=\eta}=\left.\left(\phi_{y}-\eta_{x} \phi_{x}\right)\right|_{y=\eta} .
$$

Then, it follows from (7) that $\partial_{t} \eta=G(\eta) \psi$. Directly from (4) we infer that

$$
\begin{aligned}
& \partial_{t} \psi+g \eta+N(\eta) \psi+g \eta=-P_{e x t}, \quad \text { where } \\
& N(\eta) \psi=\left.\mathcal{N}\right|_{y=\eta} \quad \text { with } \quad \mathcal{N}=\frac{1}{2} \phi_{x}^{2}-\frac{1}{2} \phi_{y}^{2}+\eta_{x} \phi_{x} \phi_{y} .
\end{aligned}
$$

With these notations, the water-wave system reads

$$
\left\{\begin{array}{l}
\partial_{t} \eta=G(\eta) \psi, \\
\partial_{t} \psi+g \eta+N(\eta) \psi=-P_{e x t} .
\end{array}\right.
$$

Introduce the energy $\mathcal{H}$, which is the sum of the potential and kinetic energies:

$$
\mathcal{H}(t)=\frac{g}{2} \int_{0}^{L} \eta^{2}(t, x) \mathrm{d} x+\frac{1}{2} \iint_{\Omega(t)}\left|\nabla_{x, y} \phi(t, x, y)\right|^{2} \mathrm{~d} x \mathrm{~d} y .
$$

If $P_{\text {ext }}=0$, then $\mathcal{H}(t)=\mathcal{H}(0)$ for all time. Our goal is to find $P_{\text {ext }}$ such that:

(i) the variation of the external pressure are localized in the absorbing beach:

$$
\operatorname{supp} \partial_{x} P_{e x t}(t, \cdot) \subset[L-\delta, L]
$$

where $\delta>0$ is the length of the absorbing beach,

(ii) $\mathcal{H}$ is decreasing,

(iii) there exists a positive constant $C$ such that

$$
\int_{0}^{T} \mathcal{H}(t) \mathrm{d} t \leq C \mathcal{H}(0)
$$

One deduces from (ii) and (iii) that

$$
\mathcal{H}(T) \leq \frac{1}{T} \int_{0}^{T} \mathcal{H}(t) \mathrm{d} t \leq \frac{C}{T} \mathcal{H}(0),
$$

which implies an exponential decay of the energy. Indeed, for $T \geq 2 C$, this gives $\mathcal{H}(T) \leq 2^{-1} \mathcal{H}(0)$ and hence $\mathcal{H}(n T) \leq 2^{-n} \mathcal{H}(0)$.

\footnotetext{
${ }^{1}$ When $P_{\text {ext }}=0$, it is proved in [4] that (10) always holds for smooth enough solutions. In fact the analysis in [4] is written only for the case $P_{\text {ext }}=0$. However, the argument still applies when $P_{\text {ext }} \neq 0$ provided that $\partial_{x} P_{\text {ext }}(t, x)=0$ when $x=0$ or $x=L$.
} 
2.2. Integral identity. To prove the key estimate (14), the main difficulty is to compute the integral of the energy $\mathcal{H}$. To do so, we will prove an exact integral identity, of the form

$$
\int_{0}^{T}(\mathcal{H}(t)+I(t)) \mathrm{d} t=\int_{0}^{T}(W(t)+O(t)+N(t)) \mathrm{d} t+B
$$

where the following properties hold:

- $I \geq 0$ and hence (15) gives an upper bound for $\int_{0}^{T} \mathcal{H} \mathrm{d} t$.

- $W$ depends on the pressure (if $P_{e x t}=0$ then $W=0$ ).

- $O$ is an observation term which means that it depends only on the behavior of the solutions near the wall $\{x=L\}$ (in the identity (16) below this requires to chose $m=x$ for $x \in[0, L-\delta])$.

- $B$ is of the form $B=\int_{0}^{L}(F(T, x)-F(0, x)) \mathrm{d} x$, for some function $F$. The key feature of this term is that, since it is not an integral in time, we can neglect $B$ for $T$ large enough.

- $N$ is a cubic term while the energy $\mathcal{H}$ and the terms $I, W, O, B$ are quadratic terms. This implies that, for the linearized water-wave equations, the same identity holds with $N=0$. So the only difference between the nonlinear problem and the linear one is described by $N$. Perhaps surprisingly, this term has a simple expression. Indeed, it is given by

$$
N(t)=\iint_{\Omega(t)} \rho_{x} \phi_{x} \phi_{y} \mathrm{~d} y \mathrm{~d} x-\int_{0}^{L} \frac{\rho}{2} \phi_{x}^{2}(t, x,-h) \mathrm{d} x,
$$

for some function $\rho$ depending linearly on $\eta$. A key point is that $N(t) \leq$ $\mathcal{H}(t)+I(t)$ for $\rho$ small enough.

In this paper we consider regular solutions of the water-wave system (12). We postpone the definition of a regular solution to $\S 3$ (see Definition 3.6). Let us mention that, essentially, this definition is quite general since we only require that the free surface elevation $\eta(t, x)$ is $C^{2}$ in $x$ and the velocity $\left.\nabla_{x, y} \phi\right|_{y=\eta(t, x)}$ is $C^{1}$ in $x$.

Here is our first main result.

Theorem 2.1. Let $m \in C^{\infty}([0, L])$ be such that $m(0)=m(L)=0$ and set

$$
\zeta=\partial_{x}(m \eta)-\frac{1}{4} \eta+\frac{1-m_{x}}{2} \eta, \quad \rho=(m-x) \eta_{x}+\left(\frac{5}{4}+\frac{m_{x}}{2}\right) \eta .
$$

Then, for any pressure $P_{\text {ext }}=P_{\text {ext }}(t, x)$ and any regular solution of (12) defined on the time interval $[0, T]$, there holds

$$
\begin{aligned}
\frac{1}{2} \int_{0}^{T} \mathcal{H}(t) \mathrm{d} t+\mathcal{Q}= & -\int_{0}^{T} \int_{0}^{L} P_{e x t} \zeta \mathrm{d} x \mathrm{~d} t-\left.\int_{0}^{L} \zeta \psi \mathrm{d} x\right|_{0} ^{T} \\
& +\int_{0}^{T} \int_{0}^{L}\left(\frac{1-m_{x}}{2} \psi+(x-m) \psi_{x}\right) G(\eta) \psi \mathrm{d} x \mathrm{~d} t \\
& +\int_{0}^{T} \iint_{\Omega(t)} \rho_{x} \phi_{x} \phi_{y} \mathrm{~d} y \mathrm{~d} x \mathrm{~d} t,
\end{aligned}
$$

where

$$
\mathcal{Q}=\int_{0}^{T} \int_{0}^{L}\left(\frac{h}{2}+\frac{\rho}{2}\right) \phi_{x}^{2}(t, x,-h) \mathrm{d} x \mathrm{~d} t+\frac{L}{2} \int_{0}^{T} \int_{-h}^{\eta(t, L)} \phi_{y}^{2}(t, L, y) \mathrm{d} y \mathrm{~d} t .
$$


Remark 2.2. (i) In [2] we proved a similar identity when $m(x)=x$ (assuming that $P_{\text {ext }}=0$ ). This weight does not vanish on $x=L$ and the identity proved in [2] was used to deduce only a boundary observability result. As explained in $\S 4$, one cannot exploit easily this boundary observability result to study the stabilization problem. By contrast, the previous identity will allow us to study this problem.

(ii) Assume that $P_{\text {ext }}=0$ and consider a small enough solution. Then, firstly, $\mathcal{Q} \geq 0$ and, secondly, one can absorb the term involving $\rho_{x} \phi_{x} \phi_{y}$ in the left-hand side. Since $\int_{0}^{T} \mathcal{H}(t) \mathrm{d} t=T \mathcal{H}(T)$ (since $P_{\text {ext }}=0$ ), we see that, loosely speaking, taking $T$ large enough, one can also absorb $\left.\int_{0}^{L} \zeta \psi \mathrm{d} x\right|_{0} ^{T}$ in the left-hand side (as explained in [2], to justify this argument requires some effort). Then we obtain an observability inequality, that is an estimate of the energy by means of the observation term $\int_{0}^{T} \int_{0}^{L}\left(\frac{1-m_{x}}{2} \psi+(x-m) \psi_{x}\right) G(\eta) \psi \mathrm{d} x \mathrm{~d} t$ (if $m(x)=x$ for $0 \leq x \leq L-\delta$, the latter expression depends only on the behavior of $\eta, \psi$ in the neighborhood of $\{x=L\})$. In Appendix $\mathrm{C}$ we prove another integral identity which involves another observation term.

2.3. Choice of the external pressure - Hamiltonian damping. As already mentioned, if $P_{\text {ext }}=0$ then the energy is conserved, that is $\mathcal{H}(t)=\mathcal{H}(0)$. Our goal is to find $P_{\text {ext }}$ so that the energy converges to zero.

For the approach developed in this paper, there are five simple principles which govern the choice of $P_{\text {ext }}$ :

(1) The energy $\mathcal{H}(t)$ must be decreasing.

(2) The Cauchy problem for (12) has to be well-posed.

(3) One could think that the stronger the damping, the faster the decay. However, in a somewhat counter-intuitive way, this is not the case. As will be clear in the proof, we need a bound of $P_{\text {ext }}$ in terms of the energy.

(4) Localization: we require that the derivative of the pressure $P_{\text {ext }}$ is localized in a neighborhood of $x=L$.

(5) Boundary condition: as already mentioned, to propagate the right-angle condition between the free surface and the wall (see (10)), the pressure $P_{\text {ext }}$ must satisfy $\partial_{x} P_{\text {ext }}(t, x)=0$ for $x=L$.

In this paragraph we give an expression for $P_{e x t}$ in terms of the unknowns such that the above five conditions are satisfied.

We begin by computing the work done by the pressure $P_{\text {ext }}$. In doing so, it is convenient to exploit the hamiltonian structure of the equation. Recall from CraigSulem ([16]) that $\mathcal{H}$ can be expressed as a function of $\eta$ and $\psi$,

$$
\mathcal{H}=\frac{1}{2} \int_{0}^{L}\left(g \eta^{2}+\psi G(\eta) \psi\right) \mathrm{d} x
$$

Then, as observed by Zakharov [41], the water-wave system can be written as ${ }^{2}$

$$
\left\{\begin{array}{l}
\frac{\partial \eta}{\partial t}=\frac{\delta \mathcal{H}}{\delta \psi}, \\
\frac{\partial \psi}{\partial t}=-\frac{\delta \mathcal{H}}{\delta \eta}-P_{\text {ext }} .
\end{array}\right.
$$

\footnotetext{
${ }^{2}$ The computations by Zakharov in [41] are written only for periodic waves and $P_{\text {ext }}=0$ but the argument holds also in a rectangular tank with an external pressure.
} 
Then write

$$
\frac{d \mathcal{H}}{d t}=\int\left[\frac{\delta \mathcal{H}}{\delta \eta} \frac{\partial \eta}{\partial t}+\frac{\delta \mathcal{H}}{\delta \psi} \frac{\partial \psi}{\partial t}\right] \mathrm{d} x=\int\left[\frac{\delta \mathcal{H}}{\delta \eta} \frac{\delta \mathcal{H}}{\delta \psi}-\frac{\delta \mathcal{H}}{\delta \psi} \frac{\delta \mathcal{H}}{\delta \eta}-\frac{\delta \mathcal{H}}{\delta \psi} P_{\text {ext }}\right] \mathrm{d} x,
$$

to deduce

$$
\frac{d \mathcal{H}}{d t}=-\int \frac{\delta \mathcal{H}}{\delta \psi} P_{e x t} \mathrm{~d} x=-\int_{0}^{L} \frac{\partial \eta}{\partial t} P_{e x t} \mathrm{~d} x=-\int_{0}^{L} P_{e x t} G(\eta) \psi \mathrm{d} x .
$$

This identity can be obtained directly from the definition (13) of the energy, using the equations and the Stokes' formula.

Since we want to force the energy to decrease to 0 , this suggests to chose $P_{\text {ext }}=$ $P_{\text {ext }}(t, x)$ under the form $P_{\text {ext }}=\chi G(\eta) \psi$ where $\chi \geq 0$ is a compactly supported function satisfying $\chi=1$ on a neighborhood of $x=L$. As mentioned in the introduction, this choice is widespread and we pause to discuss it. Firstly, with this choice, the principles (P1) and (P4) are clearly satisfied. The principle (P5) is also satisfied since $G(\eta) \psi=\partial_{t} \eta$ and since $\partial_{t} \eta$ satisfies the same boundary condition (10) as $\eta$. To see that (P3) also holds, write

$$
\int_{0}^{L} P_{\text {ext }}(t, x)^{2} \mathrm{~d} x=\int_{0}^{L}\left(\chi\left(\partial_{t} \eta\right)\right)^{2} \mathrm{~d} x \leq(\sup \chi) \int_{0}^{L} \partial_{t} \eta P_{\text {ext }} \mathrm{d} x=-(\sup \chi) \frac{d \mathcal{H}}{d t},
$$

where we used (19). It follows that we have the estimate

$$
\int_{0}^{T} \int_{0}^{L} P_{\text {ext }}(t, x)^{2} \mathrm{~d} x \mathrm{~d} t \leq(\sup \chi)(\mathcal{H}(0)-\mathcal{H}(T)) \leq(\sup \chi) \mathcal{H}(0) .
$$

However, we are not able to prove that the Cauchy problem for (12) is well-posed when $P_{e x t}$ is given by $\chi G(\eta) \psi$ (except for the linearized equations).

So we need to use another choice for $P_{\text {ext }}$. In this direction, we make the following elementary observation: by definition of $G(\eta) \psi$, it follows from the divergence theorem that

$$
\int_{0}^{L} P_{e x t} G(\eta) \psi \mathrm{d} x=\int_{\{y=\eta\}} P_{e x t} \phi_{n} \mathrm{~d} \sigma=\iint \nabla_{x, y} \cdot\left(P_{e x t} \nabla_{x, y} \phi\right) \mathrm{d} y \mathrm{~d} x .
$$

Since $\Delta_{x, y} \phi=0$ and since $P_{\text {ext }}$ does not depend on $y$, it follows from (19) that

$$
\frac{d \mathcal{H}}{d t}=-\int_{0}^{L}\left(\partial_{x} P_{e x t}\right) \bar{V} \mathrm{~d} x \quad \text { with } \quad \bar{V}(t, x)=\int_{-h}^{\eta(t, x)} \phi_{x}(t, x, y) \mathrm{d} y .
$$

Since we want to force $\mathcal{H}$ to decrease, we set

$$
\partial_{x} P_{e x t}(t, x)=\chi(x) \bar{V}(t, x),
$$

where $\chi \geq 0$ is a $C^{\infty}$ cut-off function satisfying $\chi=1$ on a neighborhood of $x=L$. The pressure $P_{\text {ext }}$ is defined up to a constant depending on time and to fix this constant we require that $P_{\text {ext }}(t, \cdot)$ has mean value 0 on $(0, L)$.

Clearly, with (20), the conditions (P1), (P4) and (P5) are satisfied (for (P5) we use the boundary condition $\left.\phi_{x}\right|_{x=L}=0$ to obtain that $\left.\left.\partial_{x} P_{e x t}\right|_{x=L}=0\right)$. By contrast with the previous choice, we will see in $\S 3.2$ that it is easy to prove that the Cauchy problem is well-posed, which means that the condition (P2) is now satisfied. Eventually, to see that (P3) also holds, we write

$$
\int_{0}^{L}\left(\partial_{x} P_{e x t}\right)^{2} \mathrm{~d} x=\int_{0}^{L}(\chi \bar{V})^{2} \mathrm{~d} x \leq(\sup \chi) \int_{0}^{L}\left(\partial_{x} P_{e x t}\right) \bar{V} \mathrm{~d} x=-(\sup \chi) \frac{d \mathcal{H}}{d t},
$$


which shows that

$$
\int_{0}^{T} \int_{0}^{L}\left(\partial_{x} P_{\text {ext }}(t, x)\right)^{2} \mathrm{~d} x \mathrm{~d} t \leq(\sup \chi) \mathcal{H}(0)
$$

2.4. A quantitative estimate. Our second main result gives an inequality of the form $\mathcal{H}(T) \leq(C / T) \mathcal{H}(0)$, for some constant $C$ depending on parameters which are considered fixed. As already mentioned, this will imply that, if the solution exists on time long time intervals of size $n T$ with $T \geq 2 C$, then the energy converges exponentially fast to zero, so that $\mathcal{H}(T) \leq 2^{-n} \mathcal{H}(0)$. In fact, we will obtain a weaker bound, of the form $\mathcal{H}(T) \leq(C / \sqrt{T}) \mathcal{H}(0)$.

A key feature of the water-wave problem is that the constant $C$ must depend on the frequency localization of $\eta$ and $\psi$. This can be easily understood by considering the linearized equations. Indeed, remembering that for these linear equations the dispersion relationship reads $\omega^{2}(k)=g|k|$, we see that high frequency waves propagate at a speed proportional to $|k|^{-1 / 2}$, which goes to 0 when $|k|$ goes to $+\infty$. Now think of waves generated near $\{x=0\}$. The time needed to reach the absorption layer (located near $\{x=L\}$ ) will depend on the frequency, and moreover will goes to $+\infty$ when $|k|$ goes to $+\infty$. This explains that the result depends on the frequency localization of the solutions, in sharp contrast with the study of other wave equations. This observation goes back to Reid and Russell ([36]) who studied the controllability in infinite time of the linearized equations.

The following result gives a quantitative estimate of the form $T \mathcal{H}(T) \leq C \mathcal{H}(0)$ where the constant $C$ depends on the frequency localization of the solutions. Since we consider the nonlinear equations, we cannot use Fourier analysis to measure the frequency localization of the solutions. We will consider instead some ratios between the energy and the $L^{2}$-norm of the derivatives of the unknown.

Theorem 2.3. Denote by $\delta>0$ the length of the absorbing zone. Consider two functions $\chi, m$ in $C^{\infty}([0, L])$ such that:

$$
\begin{aligned}
& 0 \leq \chi \leq 1, \quad \chi(x)=1 \text { if } x \in[L-\delta / 2, L], \quad \chi(x)=0 \text { if } x \in[0, L-\delta], \\
& m(x)=x \text { if } x \in[0, L-\delta / 2], \quad m(L)=0 .
\end{aligned}
$$

Assume that

$$
\partial_{x} P_{e x t}(t, x)=\chi(x) \int_{-h}^{\eta(t, x)} \phi_{x}(t, x, y) \mathrm{d} y \quad \text { and } \quad \int_{0}^{L} P_{e x t}(t, x) \mathrm{d} x=0,
$$

and introduce the functions

$$
\begin{aligned}
\rho & =(m-x) \eta_{x}+\left(\frac{5}{4}+\frac{m_{x}}{2}\right) \eta, \\
\Psi_{1} & =-m \psi_{x}-\frac{1}{4} \psi+\frac{1-m_{x}}{2} \psi, \\
\Psi_{2} & =\partial_{x}\left(\frac{1-m_{x}}{2} \psi+(x-m) \psi_{x}\right),
\end{aligned}
$$


and set

$$
\begin{aligned}
C(m) & =\sup _{x \in[0, L]} m(x)+\frac{L}{2} \sup _{x \in[0, L]}\left|1 / 2-m_{x}(x)\right|, \\
N_{1, T}(\psi) & =\sup _{t \in[0, T]} \frac{\left(\int \Psi_{1}(t, x)^{2} \mathrm{~d} x\right)^{1 / 2}}{\mathcal{H}(0)^{1 / 2}}, \\
N_{2, T}(\psi) & =\sup _{t \in[0, T]} \frac{\left(\int \Psi_{2}(t, x)^{2} \mathrm{~d} x\right)^{1 / 2}}{\mathcal{H}(0)^{1 / 2}} .
\end{aligned}
$$

If $\rho$ satisfies

$$
\rho(t, x) \geq-h, \quad\left|\rho_{x}(t, x)\right| \leq c<\frac{1}{2} .
$$

Then, for all $\alpha>0$ and for all regular solution of the water-wave system (12),

$$
T\left(\frac{1}{2}-c-\alpha\right) \mathcal{H}(T) \leq\left(\frac{C(m)^{2}}{2 \alpha g}+\sqrt{T} N_{2, T}(\psi)+\frac{2 \sqrt{2}}{\sqrt{g}} N_{1, T}(\psi)\right) \mathcal{H}(0) .
$$

Remark 2.4. Notice that $\left(\int \Psi_{1}(t, x)^{2} \mathrm{~d} x\right)^{1 / 2}\left(\operatorname{resp} .\left(\int \Psi_{2}(t, x)^{2} \mathrm{~d} x\right)^{1 / 2}\right)$ is bounded by the $L_{t}^{\infty}\left(H_{x}^{1}\right)$-norm (resp. $L_{t}^{\infty}\left(H_{x}^{2}\right)$-norm) of $\psi$. One may wonder if these norms can be controlled on large time intervals, so that the previous estimate implies that $\mathcal{H}(T) \leq c \mathcal{H}(0)$ with $c<1$. In [2], assuming that $P_{\text {ext }}=0$, we prove such bounds for small enough initial data. The same result holds when $P_{\text {ext }} \neq 0$ is as in (23) (the proof will be given in a separate paper where we will study the Cauchy problem). For the sake of completeness, we prove in the appendix such Sobolev estimates, uniformly in time, for the linearized equations (see Proposition A.1 and Remark A.2). So one may apply the previous result to these linear or weakly nonlinear settings. For the nonlinear problem, in general, one cannot propagate Sobolev estimates on large time intervals (blow-up can occur, see [12]). However, the previous estimates seem reasonable for the typical low or medium frequency waves generated in a wave tank.

Corollary 2.5. Consider two functions $\chi, m$ satisfying (22) and a regular solution of (12) satisfying (24), as in the previous statement. Consider an integer $N$ and a real number $\beta>2$. Assume that the solution exists on a time interval $[0, T]$ with $T=N^{\beta}$ and is such that, on that time interval, we have the estimates

$$
N_{1, T}(\psi) \leq N \text { and } N_{2, T}(\psi) \leq N .
$$

Then $\mathcal{H}(T) \leq \exp \left(-\delta N^{-2} T\right)$ for some constant $\delta$ depending only on $m$.

Proof. Let $\alpha$ be such that $c+\alpha<1 / 2$. Then, for any $1 \leq T^{\prime} \leq T$, we have the bound

$$
\mathcal{H}\left(T^{\prime}\right) \leq \frac{K N}{\sqrt{T^{\prime}}} \mathcal{H}(0)
$$

for some constant $K$ depending only on $g$ and $m$. Since the problem is time-invariant, we see that the same estimate holds when $\mathcal{H}\left(T^{\prime}\right)$ is replaced by $\mathcal{H}\left((k+1) T^{\prime}\right)$ and $\mathcal{H}(0)$ by $\mathcal{H}\left(k T^{\prime}\right)$, provided that $(k+1) T^{\prime} \leq T$. We obtain that

$$
\mathcal{H}\left(n T^{\prime}\right) \leq\left(\frac{K N}{\sqrt{T^{\prime}}}\right)^{n} \mathcal{H}(0)
$$

We conclude the proof by applying this inequality with $\left(n, T^{\prime}\right)$ such that $n$ is an integer, $T=n T^{\prime}$ and $T^{\prime} \geq(2 K N)^{2}$. 


\section{Study of the Cauchy problem}

We study here the Cauchy problem. In the first paragraph we consider the case $P_{\text {ext }}=0$. Our goal is to briefly recall from Alazard-Burq-Zuily [4] how to solve the Cauchy problem for the water-wave equations in a rectangular tank. In the second paragraph we explain how to extend this result to the case $P_{\text {ext }} \neq 0$.

3.1. The homogeneous problem. We recalled in the introduction that, for smooth enough solutions, the free surface must intersect the vertical walls of the tank orthogonally (see Section 6 in [4]). This means that $\eta_{x}=0$ for $x=0$ or $x=L$. Now observe that $\psi_{x}=\left.\left(\phi_{x}\right)\right|_{y=\eta}+\left.\left(\phi_{y}\right)\right|_{y=\eta} \eta_{x}$. Since $\phi_{x}(t, x, y)=0$ for $x=0$ or $x=L$, we conclude that $\psi_{x}=0$ for $x=0$ or $x=L$. As a consequence, both $\eta$ and $\psi$ will belong to the following spaces.

Definition 3.1. Given a real number $\sigma>3 / 2$, one denotes by $H_{e}^{\sigma}(0, L)$ the space

$$
H_{e}^{\sigma}(0, L)=\left\{v \in H^{\sigma}(0, L): v_{x}=0 \text { for } x=0 \text { or } x=L\right\},
$$

where $H^{\sigma}(0, L)$ denotes the usual Sobolev space of order $\sigma$.

We first need to study the problem

$$
\begin{array}{lll}
\Delta_{x, y} \phi=0 & \text { in } & \Omega=\{(x, y): x \in(0, L),-h<y<\eta(x)\}, \\
\phi=\psi & \text { for } & y=\eta(x), \\
\phi_{x}=0 & \text { for } & x=0 \text { or } x=L, \\
\phi_{y}=0 & \text { for } & y=-h .
\end{array}
$$

The following regularity result is important since it implies that all the computations made in the proof are meaningful (these computations are either integrations by parts or consequences of the Green's identity).

Proposition 3.2 (from [2]). If $(\eta, \psi) \in H_{e}^{\sigma}(0, L) \times H_{e}^{\sigma}(0, L)$ with $\sigma>5 / 2$, then there exists a unique variational solution to (26) which satisfies $\nabla_{x, y} \phi \in C^{1}(\bar{\Omega})$.

Since $\nabla_{x, y} \phi$ is continuous on $\bar{\Omega}$, one can define the Dirichlet to Neumann operator $G(\eta)$ by

$$
G(\eta) \psi=\left.\left(\phi_{y}\right)\right|_{y=\eta}-\left.\eta_{x}\left(\phi_{x}\right)\right|_{y=\eta}
$$

Since $\nabla_{x, y} \phi \in C^{1}(\bar{\Omega})$, it follows that $G(\eta) \psi \in C^{1}([0, L])$. In fact, one can prove the following stronger regularity result: If $(\eta, \psi) \in H_{e}^{\sigma}(0, L) \times H_{e}^{\sigma}(0, L)$ with $5 / 2<\sigma<$ $7 / 2$, then the traces $\left.\phi_{x}\right|_{y=\eta}$ and $\left.\phi_{y}\right|_{y=\eta}$ belong to $H_{e}^{\sigma-1}(0, L)$. Since $\eta_{x}$ also belongs to $H_{e}^{\sigma-1}(0, L)$, it follows from the usual product rule in Sobolev spaces that

$$
G(\eta) \psi \in H_{e}^{\sigma-1}(0, L) .
$$

Similarly, the nonlinear expression $N(\eta) \psi$ defined by

$$
N(\eta) \psi=\left.\mathcal{N}\right|_{y=\eta} \quad \text { with } \quad \mathcal{N}=\frac{1}{2} \phi_{x}^{2}-\frac{1}{2} \phi_{y}^{2}+\eta_{x} \phi_{x} \phi_{y},
$$

is well-defined and satisfies $N(\eta) \psi \in H_{e}^{\sigma-1}(0, L)$.

We now consider the Cauchy problem for the water-wave equations with $P_{\text {ext }}=0$,

$$
\left\{\begin{array}{l}
\partial_{t} \eta=G(\eta) \psi \\
\partial_{t} \psi+g \eta+N(\eta) \psi=0, \\
\left.(\eta, \psi)\right|_{t=0}=\left(\eta_{0}, \psi_{0}\right) .
\end{array}\right.
$$


Definition 3.3. We say that $(\eta, \psi)$ is a regular solution of (28) provided that, for some $\sigma>5 / 2$, one has

$$
(\eta, \psi) \in C^{0}\left([0, T] ; H_{e}^{\sigma}(0, L) \times H_{e}^{\sigma}(0, L)\right) \cap C^{1}\left([0, T] ; H_{e}^{\sigma-1}(0, L) \times H_{e}^{\sigma-1}(0, L)\right) .
$$

Remark. We require $\sigma>5 / 2$ to be in a position to use Proposition 3.2. Indeed, to justify all the computations below, we need that the gradient $\nabla_{x, y} \phi$ is $C^{1}$ up to the boundary.

The following result (proved in [4], see also [2]) asserts that the water-wave equations have regular solutions.

Proposition 3.4 (from [4]). Consider an initial data $\eta_{0}, \psi_{0}$ in $H_{e}^{s}(0, L)$ for some real number $s \in(3,7 / 2)$. There exists $T>0$ and a unique solution

$$
(\eta, \psi) \in C^{0}\left([0, T] ; H_{e}^{s-\frac{1}{2}}(0, L) \cap H_{e}^{s}(0, L)\right) \cap C^{1}\left([0, T] ; H_{e}^{s-\frac{3}{2}}(0, L) \cap H_{e}^{s-1}(0, L)\right),
$$

to the Cauchy problem (28).

Remark 3.5. One can overcome the apparent loss of $1 / 2$-derivative by working with different unknowns (see [2] for further comments). However, the above result, with a simple statement, will be enough for our purposes.

Let us briefly recall the strategy of the proof of Proposition 3.4. Consider an initial data $\eta_{0}, \psi_{0}:(0, L) \rightarrow \mathbb{R}$ in $H_{e}^{s}(0, L)$ with $s>3$. Following Boussinesq (see [10, page $37]$ ), the proof consists in extending these initial data to periodic functions, for which one can solve the Cauchy problem. Then one deduces the existence of solutions to the water-wave system in a tank by considering the restrictions of these solutions.

To obtain periodic functions we use in [4] a classical reflection/periodization procedure (with respect to the normal variable to the boundary of the tank). Notice that, in general, the even extension of a regular function on $(0, L)$ to a function defined on $(-L, L)$ is merely Lipschitz continuous (for instance one obtains $|x|$ starting from $x \mapsto x)$. Now, the main difficulty is that there is no result which allows to handle Lipschitz free surface. However, when the free surface intersects the walls with a right angle, the reflected domain enjoys additional smoothness (namely up to $C^{3}$ ), which is enough to solve the Cauchy problem (this raises many other questions and we refer to [4] for more details).

3.2. The inhomogeneous problem. We now consider the inhomogeneous problem and assume that $P_{\text {ext }}$ satisfies

$$
\partial_{x} P_{e x t}(t, x)=\chi(x) \bar{V}(t, x) \quad \text { with } \quad \bar{V}(t, x)=\int_{-h}^{\eta(t, x)} \phi_{x}(t, x, y) \mathrm{d} y,
$$

and where $\chi$ is a $C^{\infty}$ cut-off function. The pressure $P_{\text {ext }}$ is defined up to a timedependent function and to fix $P_{\text {ext }}$ we require that $P_{\text {ext }}$ has mean value 0 on $(0, L)$.

Definition 3.6. As above, we say that $(\eta, \psi)$ is a regular solution to the water-wave equations (see (30)) provided that, for some $\sigma>5 / 2$, one has

$$
(\eta, \psi) \in C^{0}\left([0, T] ; H_{e}^{\sigma}(0, L) \times H_{e}^{\sigma}(0, L)\right) \cap C^{1}\left([0, T] ; H_{e}^{\sigma-1}(0, L) \times H_{e}^{\sigma-1}(0, L)\right) .
$$

Hereafter, we assume that the initial data satisfies the so-called Taylor sign condition. The Taylor sign condition states that the pressure increases going from the air into the fluid domain. It is always satisfied when there is no pressure (see [40, 25]). 
Proposition 3.7. Consider an initial data $\eta_{0}, \psi_{0}$ in $H_{e}^{s}(0, L)$ for some real number $s \in(3,7 / 2)$, satisfying the Taylor sign condition. There exist $T>0$ and a unique solution

$$
(\eta, \psi) \in C^{0}\left([0, T] ; H_{e}^{s-\frac{1}{2}}(0, L) \times H_{e}^{s}(0, L)\right) \cap C^{1}\left([0, T] ; H_{e}^{s-\frac{3}{2}}(0, L) \times H_{e}^{s-1}(0, L)\right),
$$

to the Cauchy problem

$$
\left\{\begin{array}{l}
\partial_{t} \eta=G(\eta) \psi \\
\partial_{t} \psi+g \eta+N(\eta) \psi=P_{e x t} \\
\left.(\eta, \psi)\right|_{t=0}=\left(\eta_{0}, \psi_{0}\right)
\end{array}\right.
$$

where $P_{\text {ext }}$ is given by (29).

We claim that this result follows from the proof of Proposition 3.4. To see this, we have to check two different properties.

The first remark to be made is that the previous reflection/periodization procedure applies with a source term $P_{\text {ext }}$ provided that $P_{\text {ext }}$ has the same parity as $\psi$. Here, since after reflection, $\eta$ and $\phi$ are even in $x$, the function $\bar{V}$ is odd in $x$ and hence $P_{\text {ext }}$ is even in $x$. Since $\psi$ is also even in $x$, we verify that $P_{\text {ext }}$ and $\psi$ have the same parity.

Secondly, we need to know the effect of $P_{\text {ext }}$ on the Sobolev energy estimates used in the analysis of the Cauchy problem. The key point is that $P_{e x t}$ is a lower order term which can be handled as a source term in all energy estimates. This is where we use in a crucial way the choice of the pressure term. Indeed, we claim that

$$
\partial_{x} P_{e x t}(t, x)=-\chi(x) \int_{0}^{x} G(\eta) \psi(t, X) \mathrm{d} X .
$$

To see this, recall that $\Delta_{x, y} \phi=0$ and $\phi_{n}=0$ for $x=0$ and $y=-h$. With $Q(x)=\{(X, y): X \in[0, x],-h \leq y \leq \eta(X)\}$, the divergence theorem implies that

$$
0=\int_{\partial Q(x)} \phi_{n} \mathrm{~d} \sigma=\int_{-h}^{\eta(t, x)} \phi_{x}(t, x, y) \mathrm{d} y+\int_{\substack{y=\eta(X) \\ X \in[0, x]}} \phi_{n} \mathrm{~d} \sigma .
$$

This yields the well-known formula (see $\S 3.5$ in [25])

$$
\bar{V}(t, x)+\int_{0}^{x} G(\eta) \psi(t, X) \mathrm{d} X=0
$$

which implies (31). Now, if $(\eta, \psi) \in H_{e}^{s-\frac{1}{2}}(0, L) \times H_{e}^{s-\frac{1}{2}}(0, L)$ with $3<s<7 / 2$, we have already recalled that $G(\eta) \psi$ belongs to $H_{e}^{s-3 / 2}(0, L)$. The previous formula implies that $P_{\text {ext }}$ belongs to $H_{e}^{s+1 / 2}(0, L)$. It turns out that this is exactly the regularity needed to consider $P_{\text {ext }}$ as a source term ${ }^{3}$.

\footnotetext{
${ }^{3}$ For the sake of conciseness, we will not enter into the details. We mention the recent work by Mélinand [31] where the author studies several questions about the water-wave problem with a source term. However, the well-posedness result in [31] applies for smoother initial data which is insufficient to prove Proposition 3.7. Nevertheless, an inspection of the analysis in [4] shows that, for any $s>3$, one can consider a source term $P_{\text {ext }}$ provided that $P_{\text {ext }} \in L^{1}\left(0, T ; H^{s+1 / 2}(0, L)\right)$.
} 


\section{Strategy of the Proof: Introduction to the Multiplier method}

The control theory of wave equations is well developed and many techniques have been introduced (microlocal analysis, Carleman estimates...). In this paper, we use the multiplier method. The key point is that this method allows us to work directly at the level of the nonlinear equations.

For the sake of readability, we begin by recalling some well-known results for the linear wave equation

$$
\partial_{t}^{2} u-\Delta u=0 \quad \text { in } \Omega \subset \mathbb{R}^{n},\left.\quad u\right|_{\partial \Omega}=0 .
$$

The multiplier method, introduced by Morawetz, consists in multiplying the equations by $m(x) \cdot \nabla u(t, x)$, for some well-chosen function $m$, and to integrate by parts in space and time. For instance, by considering a smooth extension $m: \Omega \rightarrow \mathbb{R}^{n}$ of the normal $\nu(x)$ to the boundary $\partial \Omega$, one obtains

$$
\int_{0}^{T} \int_{\partial \Omega}\left(\partial_{n} u\right)^{2} \mathrm{~d} \sigma \mathrm{d} t \leq K(T) \mathcal{E}(u) \text { where } \mathcal{E}(u):=\|u(0, \cdot)\|_{H_{0}^{1}(\Omega)}^{2}+\left\|\partial_{t} u(0, \cdot)\right\|_{L^{2}(\Omega)}^{2} .
$$

This is the so-called hidden regularity property. The name comes from the fact that, using energy estimates, one controls only the $C^{0}\left([0, T] ; L^{2}(\Omega)\right)$-norm of $\nabla_{x} u$ by means of the right-hand side of (34), which is insufficient to control the left-hand side of (34) by means of classical trace theorems.

Another key estimate is the so-called boundary observability inequality, which is, compared to (34), a reverse inequality where one can bound the norms of the initial data by the integral of $\partial_{n} u$ restricted to a domain $\Gamma_{0} \subset \partial \Omega$. Such an inequality can be obtained by the multiplier method applied in this way: fix $x_{0} \in \mathbb{R}^{n}$ and set

$$
\Gamma\left(x_{0}\right)=\left\{x \in \partial \Omega,\left(x-x_{0}\right) \cdot \nu(x)>0\right\}, \quad T\left(x_{0}\right)=2 \max _{x \in \bar{\Omega}}\left|x-x_{0}\right| .
$$

Then, multiplying the equation by $\left(x-x_{0}\right) \cdot \nabla u$ and integrating by parts, we get that, for $T>T\left(x_{0}\right)$,

$$
\left(T-T\left(x_{0}\right)\right) \mathcal{E}(u) \leq \frac{T\left(x_{0}\right)}{2} \int_{0}^{T} \int_{\Gamma\left(x_{0}\right)}\left(\partial_{n} u\right)^{2} \mathrm{~d} \sigma \mathrm{d} t .
$$

For more details about the previous two inequalities, we refer the reader to the SIAM Review article by Lions [28] and the books by Komornik [24], Micu and Zuazua [32], Tucsnak and Weiss [39] and the lecture notes by Alabau-Boussouira in [1].

Now consider a domain $\omega$ surrounding $\Gamma\left(x_{0}\right)$. The proof of the hidden regularity property (34) allows us to bound the right-hand side in (35) by the sum of $C_{1} \mathcal{E}$ (where $C_{1}$ is independent of time) and the integral of $|\nabla u|^{2}$ on $(0, T) \times \omega$. Then, for $T$ large enough, one can absorb the term $C_{1} \mathcal{E}$ in the left-hand side of (35) to deduce the following internal observability inequality:

$$
\mathcal{E}(u) \leq C(T) \int_{0}^{T} \int_{\omega}|\nabla u|^{2} \mathrm{~d} x \mathrm{~d} t
$$

This inequality can be used to obtain directly a stabilization result for the following damped wave equation

$$
\partial_{t}^{2} v-\Delta v+a(x) \partial_{t} v=0 \quad \text { in } \Omega \subset \mathbb{R}^{n},\left.\quad v\right|_{\partial \Omega}=0,
$$


where $a \in C_{0}^{\infty}(\Omega)$ is a non-negative function satisfying $a(x)=1$ for $x$ in $\omega \subset \subset \Omega$. One can write $v$ as $v=u+w$ where $u$ and $w$ are given by solving

$$
\begin{aligned}
& \partial_{t}^{2} u-\Delta u=0 \quad \text { in } \Omega \subset \mathbb{R}^{n},\left.\quad u\right|_{\partial \Omega}=0, \\
& \partial_{t}^{2} w-\Delta w+a(x) \partial_{t} w=-a(x) \partial_{t} u \quad \text { in } \Omega \subset \mathbb{R}^{n},\left.\quad w\right|_{\partial \Omega}=0, \\
& u(0, \cdot)=v(0, \cdot), \quad \partial_{t} u(t, 0)=\partial_{t} v(0, \cdot) ; \quad w(0, \cdot)=0, \quad \partial_{t} w(t, 0)=0 .
\end{aligned}
$$

Using the internal observability inequality for $u$ and a straightforward estimate for $w$ based on the Duhamel formula, one can deduce that $\mathcal{E}(v)(t) \leq c e^{-c^{\prime} t}$ for some positive constants $c, c^{\prime}$.

Similar results are known for many other wave equations and we only mention the paper by Machtyngier [29] (see also [30]) for the Schrödinger equation $i \partial_{t} u+\Delta u=0$. Biccari [7] introduced recently the use of the multiplier method to analyze the interior controllability problem for the fractional Schrödinger equation $i \partial_{t} u+(-\Delta)^{s} u=0$ with $s \geq 1 / 2$ in a $C^{1,1}$ bounded domain with Dirichlet boundary condition. The key difference between the Schrödinger equation $(s=1)$ and the fractional equation (for $1 / 2 \leq s<1$ ) is that the latter is nonlocal. This is a source of difficulty since one seeks an observability result involving integrals over small localized domains. In particular, a key technical difference is that one needs to compute $\int(-\Delta)^{s} u(x \cdot \nabla u) \mathrm{d} x$. The result is called a Pohozaev identity, since Pohozaev introduce the use of the multiplier $x \cdot \nabla u$ to study properties of elliptic equations (we refer to [37] for such identities for fractional Laplacians).

In our previous paper [2], we introduce the use of the multiplier method to study the gravity water-wave equations. To compare with the study by Biccari, notice that the linearized gravity water-wave equations can be written as $i \partial_{t} u+(-\Delta)^{s} u=0$ with $s=1 / 4$ and hence the assumption $s \geq 1 / 2$ does not hold. This is a key feature of the problem since the group velocity is $|\xi|^{2 s-1}$ and hence, for $s<1 / 2$, high frequency waves propagate at a speed which goes to 0 when $|\xi|$ goes to $+\infty$. Also, in $[7,37]$, the authors consider the case where $\Delta$ is the Laplacian with Dirichlet boundary condition while we consider periodic functions here. More importantly, the main difficulty in [2] or in the present paper is that the equations are nonlinear. In particular, we need a Pohozaev identity for $\int(G(\eta) \psi)(x \cdot \nabla \psi) \mathrm{d} x$ where $G(\eta)$ is an operator with variable coefficients.

Let us now explain the main difficulties one has to cope with to stabilize the waterwave equations. Firstly, one cannot decouple the problem of the observability and the question of the stabilization. Compared to what is done for the wave equation (see (37)), since the water-wave system is quasi-linear, one cannot write the solution as the sum of the two different problems. This means that one cannot assume that $P_{\text {ext }}=0$ for the purpose of proving observability. Another difficulty is that we do not know how to deduce an internal observability inequality from a boundary observability inequality (for the wave equation or the Schrödinger equation, as we recalled above, this is possible thanks to a hidden regularity result). To overcome these two problems, guided by the lectures notes by Alabau-Boussouira ([1]), we prove directly an internal observability result for the water-wave system by considering a multiplier $m(x) \partial_{x}$ with $m(x)=x \kappa(x)$ where $\kappa$ is a cut-off function satisfying $\kappa(x)=1$ for $0 \leq x \leq L-\delta$ and $\kappa(x)=0$ for $L-\delta / 2 \leq x \leq L$. 


\section{Proof of Theorem 2.1}

The proof is in four steps.

Notation. We write simply

$$
\int d x, \int d y, \int d t
$$

as shorthand notations for, respectively,

$$
\int_{0}^{L} d x, \quad \int_{-h}^{\eta(t, x)} d y, \quad \int_{0}^{T} d t
$$

Step 1 : the multiplier method. To estimate $\int \mathcal{H}(t) \mathrm{d} t$, we will use in a crucial way the unknown

$$
\Theta:=-\eta \partial_{t} \psi-\frac{g}{2} \eta^{2}
$$

In Appendix B, we will see that this function is related to Luke's variational principle. This observation explains that we will be able to compare $\iint \Theta \mathrm{d} x \mathrm{~d} t$ and $\int \mathcal{H}(t) \mathrm{d} t$. The function $\Theta$ was introduced in [2] for the purpose of proving a boundary observability result. In that reference, we used the weight $m(x)=x$. Now, for a general weight $m(x)$, to obtain an identity for $\iint \Theta \mathrm{d} x \mathrm{~d} t$ we proceed in a different way. We write

$$
\iint \Theta \mathrm{d} x \mathrm{~d} t=\iint m_{x} \Theta \mathrm{d} x \mathrm{~d} t+\iint\left(1-m_{x}\right) \Theta \mathrm{d} x \mathrm{~d} t .
$$

The second term in the right-hand side is an observation term. Indeed, if $m(x)=$ $x \kappa(x)$ where $\kappa(x)=1$ in $[0, L-\delta]$ and $\kappa(x)=0$ in $[L-\delta / 2, L]$, then $\left(1-m_{x}\right) \Theta$ depends only on the behavior of $\eta$ and $\psi$ in a neighborhood of $x=L$. So the key point is to obtain an identity for $\iint m_{x} \Theta \mathrm{d} x \mathrm{~d} t$. This is the purpose of the following lemma.

Lemma 5.1. Consider a smooth solution of the water-wave system and a smooth function $m:[0, L] \rightarrow \mathbb{R}$ satisfying $m(0)=m(L)=0$. Then one has

$$
\iint m_{x} \Theta \mathrm{d} x \mathrm{~d} t+R_{a}=-\left.\int \partial_{x}(m \eta) \psi \mathrm{d} x\right|_{0} ^{T}-\iint P_{e x t} m \eta_{x} \mathrm{~d} x \mathrm{~d} t
$$

where

$$
R_{a}=\iint(G(\eta) \psi) m \psi_{x} \mathrm{~d} x \mathrm{~d} t+\iint(N(\eta) \psi) m \eta_{x} \mathrm{~d} x \mathrm{~d} t .
$$

Proof. The proof is based on the multiplier method applied in the following way: instead of multiplying the equations by $\left(m \partial_{x} \eta, m \partial_{x} \psi\right)$, we set

$$
A:=\iint\left[\left(\partial_{t} \eta\right)\left(m \partial_{x} \psi\right)-\left(\partial_{t} \psi\right)\left(m \partial_{x} \eta\right)\right] \mathrm{d} x \mathrm{~d} t,
$$

and we compute $A$ in two different ways. Then the wanted identity will be deduced by comparing the two results.

First computation. Since $m(0)=m(L)=0$, directly from the definition of $A$, using integration by parts in space and time, one has

$$
A=\left.\int m \eta \psi_{x} \mathrm{~d} x\right|_{0} ^{T}+\iint m_{x} \eta \partial_{t} \psi \mathrm{d} x \mathrm{~d} t
$$


Since $m(0)=m(L)=0$ one can further integrate by parts in $x$ in the first term to obtain

$$
A=-\left.\int \partial_{x}(m \eta) \psi \mathrm{d} x\right|_{0} ^{T}+\iint m_{x} \eta \partial_{t} \psi \mathrm{d} x \mathrm{~d} t
$$

Second computation. We simply compute $A$ by replacing $\partial_{t} \eta$ and $\partial_{t} \psi$ by the expressions given by System (12). We find that

$$
A=\iint\left(P_{e x t}+g \eta\right) m \eta_{x} \mathrm{~d} x+R_{a}
$$

where $R_{a}$ is given by (38). On the other hand, since $m(0)=m(L)=0$, integrating by parts, we obtain

$$
-\int g \eta m \eta_{x} \mathrm{~d} x=\frac{1}{2} \int g m_{x} \eta^{2} \mathrm{~d} x
$$

By combining this identity with (40), it follows that

$$
A=-\frac{1}{2} \int g m_{x} \eta^{2} \mathrm{~d} x+\iint P_{e x t} m \eta_{x} \mathrm{~d} x+R_{a} .
$$

Then, by comparing the previous identity with (39) we conclude the proof.

Step 2: equipartition of the energy. Introduce the average in time kinetic (resp. potential) energy denoted by $A_{K}$ (resp. $\left.A_{P}\right)$. By definition,

$$
A_{K}=\frac{1}{2} \iint \psi G(\eta) \psi \mathrm{d} x \mathrm{~d} t, \quad A_{P}=\frac{g}{2} \iint \eta^{2} \mathrm{~d} x \mathrm{~d} t
$$

and we have

$$
\int \mathcal{H}(t) \mathrm{d} t=A_{K}+A_{P}
$$

The analysis below relies heavily on the idea of comparing $A_{K}$ and $A_{P}$. We will see that one has equipartition of the energy, which means that the difference between these two quantities can be handled as a remainder term. We will not only compare $A_{K}$ and $A_{P}$ but also some localized versions where we add an extra factor $\chi=\chi(x)$ in the integrals.

Lemma 5.2. For any smooth function $\chi=\chi(x)$, there holds

$$
\begin{aligned}
\frac{g}{2} \iint \chi \eta^{2} \mathrm{~d} x \mathrm{~d} t= & \frac{1}{2} \iint \chi \psi G(\eta) \psi \mathrm{d} x \mathrm{~d} t \\
& -\frac{1}{2} \iint \chi \eta P_{e x t} \mathrm{~d} x \mathrm{~d} t-\left.\frac{1}{2} \int \chi \eta \psi \mathrm{d} x\right|_{0} ^{T} \\
& -\frac{1}{2} \iint \chi \eta N(\eta) \psi \mathrm{d} x \mathrm{~d} t
\end{aligned}
$$

In particular, with $\chi=1$, one has

$$
A_{K}-A_{P}=\frac{1}{2} \iint \eta P_{e x t} \mathrm{~d} x \mathrm{~d} t+R_{b}+\left.\frac{1}{2} \int \eta \psi \mathrm{d} x\right|_{0} ^{T},
$$

where

$$
R_{b}=\frac{1}{2} \iint \eta N(\eta) \psi \mathrm{d} x \mathrm{~d} t
$$


Proof. Using $\partial_{t} \eta=G(\eta) \psi$ and integrating by parts, we find that

$$
\begin{aligned}
\frac{1}{2} \iint \chi \psi G(\eta) \psi \mathrm{d} x \mathrm{~d} t-\frac{g}{2} \iint \chi \eta^{2} \mathrm{~d} x \mathrm{~d} t & =\frac{1}{2} \iint \chi\left[\psi\left(\partial_{t} \eta\right)-g \eta^{2}\right] \mathrm{d} x \mathrm{~d} t \\
& =\frac{1}{2} \iint \chi\left[-\eta\left(\partial_{t} \psi+g \eta\right)\right] \mathrm{d} x \mathrm{~d} t \\
& +\left.\frac{1}{2} \int \chi \eta \psi \mathrm{d} x\right|_{0} ^{T} .
\end{aligned}
$$

So (42) follows from the equations (12) for $\psi$.

By combining the previous identities, we will deduce the following lemma.

Lemma 5.3. Set

There holds

$$
\zeta=\partial_{x}(m \eta)-\frac{1}{4} \eta+\frac{1-m_{x}}{2} \eta
$$

$$
\begin{aligned}
\frac{1}{2} \int \mathcal{H}(t) \mathrm{d} t= & -\iint P_{\text {ext }} \zeta \mathrm{d} x \mathrm{~d} t \\
& +\frac{1}{2} \iint\left(1-m_{x}\right) \psi G(\eta) \psi \mathrm{d} x \mathrm{~d} t \\
& -\left.\int \zeta \psi \mathrm{d} x\right|_{0} ^{T} \\
& -\iint(G(\eta) \psi) m \psi_{x} \mathrm{~d} x \mathrm{~d} t \\
& -\iint \zeta(N(\eta) \psi) \mathrm{d} x \mathrm{~d} t .
\end{aligned}
$$

Proof. Recall that $\Theta=-\eta \partial_{t} \psi-\frac{g}{2} \eta^{2}$. Then, using the equation (12) for $\psi$, we get

$$
\Theta=-\eta\left(\partial_{t} \psi+g \eta\right)+\frac{g}{2} \eta^{2}=\eta\left(P_{e x t}+N(\eta) \psi\right)+\frac{g}{2} \eta^{2},
$$

which implies that

$$
\iint m_{x} \Theta \mathrm{d} x \mathrm{~d} t=\frac{g}{2} \iint m_{x} \eta^{2} \mathrm{~d} x \mathrm{~d} t+\iint P_{\text {ext }} m_{x} \eta \mathrm{d} x \mathrm{~d} t+R_{c}
$$

where $R_{c}$ is given by

$$
R_{c}=\iint m_{x} \eta(N(\eta) \psi) \mathrm{d} x \mathrm{~d} t
$$

Now recall from Lemma 5.1 that

$$
\iint m_{x} \Theta \mathrm{d} x \mathrm{~d} t+R_{a}=\left.\int m \eta \psi_{x} \mathrm{~d} x\right|_{0} ^{T}-\iint P_{e x t} m \eta_{x} \mathrm{~d} x \mathrm{~d} t .
$$

Then, it follows from (45) that

$$
\frac{g}{2} \iint m_{x} \eta^{2} \mathrm{~d} x \mathrm{~d} t+R_{a}+R_{c}=\left.\int m \eta \psi_{x} \mathrm{~d} x\right|_{0} ^{T}-\iint P_{e x t} \partial_{x}(m \eta) \mathrm{d} x \mathrm{~d} t .
$$

We then split the coefficient $m_{x}$ in the left-hand side as $m_{x}=1+\left(m_{x}-1\right)$ to obtain

$$
\begin{aligned}
\frac{g}{2} \iint \eta^{2} \mathrm{~d} x \mathrm{~d} t+R_{a}+R_{c}= & \left.\int m \eta \psi_{x} \mathrm{~d} x\right|_{0} ^{T}-\iint P_{e x t} \partial_{x}(m \eta) \mathrm{d} x \mathrm{~d} t \\
& +\frac{g}{2} \iint\left(1-m_{x}\right) \eta^{2} \mathrm{~d} x \mathrm{~d} t .
\end{aligned}
$$


On the other hand, it follows from (41) and (43) that

$$
\begin{aligned}
\frac{g}{2} \iint \eta^{2} \mathrm{~d} x \mathrm{~d} t & =A_{P}=\frac{1}{2}\left(A_{K}+A_{P}\right)+\frac{1}{2}\left(A_{P}-A_{K}\right) \\
& =\frac{1}{2} \int \mathcal{H}(t) \mathrm{d} t-\frac{1}{4} \iint \eta P_{\text {ext }} \mathrm{d} x \mathrm{~d} t-\frac{1}{2} R_{b}-\left.\frac{1}{4} \int \eta \psi \mathrm{d} x\right|_{0} ^{T} .
\end{aligned}
$$

By combining the previous results, we get that

$$
\begin{aligned}
\frac{1}{2} \int \mathcal{H}(t) \mathrm{d} t= & -\iint P_{\text {ext }}\left(\partial_{x}(m \eta)-\frac{1}{4} \eta\right) \mathrm{d} x \mathrm{~d} t \\
& +\frac{g}{2} \iint\left(1-m_{x}\right) \eta^{2} \mathrm{~d} x \mathrm{~d} t \\
& -\iint(G(\eta) \psi) m \psi_{x} \mathrm{~d} x \mathrm{~d} t \\
& -\left.\int\left(\partial_{x}(m \eta)-\frac{1}{4} \eta\right) \psi \mathrm{d} x\right|_{0} ^{T} \\
& -\iint\left(\partial_{x}(m \eta)-\frac{1}{4} \eta\right) N(\eta) \psi \mathrm{d} x \mathrm{~d} t .
\end{aligned}
$$

On the other hand, it follows from (42) that

$$
\begin{aligned}
\frac{g}{2} \iint\left(1-m_{x}\right) \eta^{2} \mathrm{~d} x \mathrm{~d} t= & \frac{1}{2} \iint\left(1-m_{x}\right) \psi G(\eta) \psi \mathrm{d} x \mathrm{~d} t \\
& -\frac{1}{2} \iint\left(1-m_{x}\right) \eta P_{e x t} \mathrm{~d} x \mathrm{~d} t-\left.\frac{1}{2} \int\left(1-m_{x}\right) \eta \psi \mathrm{d} x\right|_{0} ^{T} \\
& -\frac{1}{2} \iint\left(1-m_{x}\right) \eta N(\eta) \psi \mathrm{d} x \mathrm{~d} t
\end{aligned}
$$

By plugging (48) in (47), we obtain the desired identity (44).

Step 3: a Pohozaev identity. To complete the proof of the theorem, it remains to study the last two terms in the right-hand side of (44). We begin with the last but one term

$$
\int(G(\eta) \psi) m \psi_{x} \mathrm{~d} x
$$

To handle this term, we split it into two terms in order to obtain an expression which make appear a positive term through a Pohozaev identity. So we write

$$
\int(G(\eta) \psi) m \psi_{x} \mathrm{~d} x=\int(G(\eta) \psi) x \psi_{x} \mathrm{~d} x+\int(G(\eta) \psi)(m-x) \psi_{x} \mathrm{~d} x .
$$

We now use the following Pohozaev identity proved in [2].

Lemma 5.4 (from [2]). One has

$$
\int(G(\eta) \psi) x \psi_{x} \mathrm{~d} x=\Sigma+\int\left(\eta-x \eta_{x}\right)(N(\eta) \psi) \mathrm{d} x,
$$

where $\Sigma=\Sigma(t)$ is a positive term given by

$$
\Sigma(t)=\frac{h}{2} \int_{0}^{L} \phi_{x}^{2}(t, x,-h) \mathrm{d} x+\frac{L}{2} \int_{-h}^{\eta(t, L)} \phi_{y}^{2}(t, L, y) \mathrm{d} y .
$$


Observe that, by integrating in time, we obtain (17) with $\mathcal{Q}=\int_{0}^{T} Q(t) \mathrm{d} t$. By so doing, we end up with

$$
\begin{aligned}
\frac{1}{2} \int \mathcal{H}(t) \mathrm{d} t+\int \Sigma(t) \mathrm{d} t= & -\iint P_{\text {ext }} \zeta \mathrm{d} x \mathrm{~d} t \\
& +\iint\left(\frac{1-m_{x}}{2} \psi+(x-m) \psi_{x}\right) G(\eta) \psi \mathrm{d} x \mathrm{~d} t \\
& -\left.\int \zeta \psi \mathrm{d} x\right|_{0} ^{T} \\
& -\iint \rho N(\eta) \psi \mathrm{d} x \mathrm{~d} t .
\end{aligned}
$$

where the coefficient $\rho$ in the last term is given by

$$
\rho=\zeta+\eta-x \eta_{x}=(m-x) \eta_{x}+\left(\frac{5}{4}+\frac{m_{x}}{2}\right) \eta .
$$

Step 4: computation of the remainder term. In view of a possible application to the stabilization problem, the previous identity (51) is not sufficient since one cannot control a priori the last term $\int \rho N(\eta) \psi \mathrm{d} x$ by means of the energy. Indeed,

$$
N(\eta) \psi=\left.\mathcal{N}\right|_{y=\eta} \quad \text { with } \quad \mathcal{N}=\frac{1}{2} \phi_{x}^{2}-\frac{1}{2} \phi_{y}^{2}+\eta_{x} \phi_{x} \phi_{y}
$$

and clearly one cannot simply use the previous definition to bound $N(\eta) \psi$ by $K \iint\left|\nabla_{x, y} \phi\right|^{2} \mathrm{~d} y \mathrm{~d} x$ using only the trace theorem. However, as in [2], inspired by the analysis done by Benjamin and Olver ([6]) of the conservation laws for water waves, one can rewrite $\int \rho N(\eta) \psi \mathrm{d} x$ as the sum of two terms which can be controlled either by the energy or by the positive term $\Sigma$ given by the Pohozaev identity.

Lemma 5.5. There holds

$$
\int \rho N(\eta) \psi \mathrm{d} x=-\iint \rho_{x} \phi_{x} \phi_{y} \mathrm{~d} y \mathrm{~d} x+\left.\frac{1}{2} \int \rho \phi_{x}^{2}\right|_{y=-h} \mathrm{~d} x .
$$

Proof. This result will be obtained by writing $\int \rho N(\eta) \psi \mathrm{d} x$ under the form

$$
\iint u(t, x, \eta(t, x)) \mathrm{d} x \mathrm{~d} t+\iint f(t, x, \eta(t, x)) \eta_{x}(t, x) \mathrm{d} x \mathrm{~d} t,
$$

together with an application of the following elementary identity: for any functions $u=u(x, y)$ and $f=f(x, y)$ with $\left.f\right|_{x=0}=\left.f\right|_{x=L}=0$, one has

$$
\int u(x, \eta(x)) \mathrm{d} x+\int f(x, \eta) \eta_{x} \mathrm{~d} x=\iint\left(u_{y}-f_{x}\right) \mathrm{d} y \mathrm{~d} x+\int u(x,-h) \mathrm{d} x .
$$

Indeed,

$$
\int_{0}^{L} u(x, \eta(x)) \mathrm{d} x=\int_{0}^{L} \int_{-h}^{\eta(x)} u_{y}(x, y) \mathrm{d} y+\int_{0}^{L} u(x,-h) \mathrm{d} x
$$

and

$$
\int_{0}^{L} f(x, \eta) \eta_{x} \mathrm{~d} x+\int_{0}^{L} \int_{-h}^{\eta(x)} f_{x} \mathrm{~d} y \mathrm{~d} x=\left.\int_{-h}^{\eta} f \mathrm{~d} y \mathrm{~d} x\right|_{x=0} ^{x=L}=0 .
$$

Now, by definition of $N(\eta) \psi$, we have

$$
\int \rho N(\eta) \psi \mathrm{d} x=\int u(x, \eta) \mathrm{d} x+\int f(x, \eta) \eta_{x} \mathrm{~d} x,
$$


with

$$
u(x, y)=\frac{1}{2} \rho\left(\phi_{x}^{2}-\phi_{y}^{2}\right), \quad f(x, y)=\rho \phi_{x} \phi_{y} .
$$

Since $\left.f\right|_{x=0}=\left.f\right|_{x=L}=0$ and

$$
\left.u\right|_{y=-h}=\left.\frac{1}{2} \rho \phi_{x}^{2}\right|_{y=-h}, \quad u_{y}-f_{x}=-\rho_{x} \phi_{x} \phi_{y},
$$

the desired result (52) follows from (53).

By plugging this result into (51), we complete the proof of Theorem 2.1.

\section{Proof of Proposition 2.3}

We want to prove an inequality of the form

$$
T \mathcal{H}(T) \leq C \mathcal{H}(0)
$$

where $C$ is as given by the right-hand side of (25). To do so, we will prove that

$$
\int_{0}^{T} \mathcal{H}(t) \mathrm{d} t \leq C \mathcal{H}(0)
$$

Then the desired bound (54) will be deduced from (55) and the fact that the energy is decreasing, so that $T \mathcal{H}(T) \leq \int_{0}^{T} \mathcal{H}(t) \mathrm{d} t$.

Lemma 6.1. Assume that $\rho$ satisfies

$$
\rho(t, x) \geq-h, \quad\left|\rho_{x}(t, x)\right| \leq c<\frac{1}{2} .
$$

Then

$$
\begin{aligned}
\left(\frac{1}{2}-c\right) \int_{0}^{T} \mathcal{H}(t) \mathrm{d} t \leq & -\int_{0}^{T} \int_{0}^{L} P_{e x t} \zeta \mathrm{d} x \mathrm{~d} t-\left.\int_{0}^{L} \zeta \psi \mathrm{d} x\right|_{0} ^{T} \\
& +\int_{0}^{T} \int_{0}^{L}\left(\frac{1-m_{x}}{2} \psi+(x-m) \psi_{x}\right) G(\eta) \psi \mathrm{d} x \mathrm{~d} t .
\end{aligned}
$$

Proof. The assumptions on $\rho$ imply that $\mathcal{Q} \geq 0$ as well as the estimate

$$
\int_{0}^{T} \iint_{\Omega(t)} \rho_{x} \phi_{x} \phi_{y} \mathrm{~d} y \mathrm{~d} x \mathrm{~d} t \leq \frac{c}{2} \int_{0}^{T} \iint_{\Omega(t)}\left(\phi_{x}^{2}+\phi_{y}^{2}\right) \mathrm{d} y \mathrm{~d} x \mathrm{~d} t \leq c \int_{0}^{T} \mathcal{H}(t) \mathrm{d} t .
$$

The wanted inequality then immediately follows from Theorem 2.1.

Notation. We use the notations

$$
\|f\|_{L^{2}}=\left(\int_{0}^{L} f(x)^{2} \mathrm{~d} x\right)^{1 / 2}, \quad\|f\|_{L^{\infty}}=\sup _{x \in[0, L]}|f(x)| .
$$

Lemma 6.2. For any $\alpha>0$,

$$
\begin{aligned}
& -\int_{0}^{T} \int_{0}^{L} P_{e x t} \zeta \mathrm{d} x \mathrm{~d} t+\int_{0}^{T} \int_{0}^{L}\left(\frac{1-m_{x}}{2} \psi+(x-m) \psi_{x}\right) G(\eta) \psi \mathrm{d} x \mathrm{~d} t \\
& \leq \frac{C(m)^{2}}{2 \alpha g} \int_{0}^{T}\left\|\partial_{x} P_{e x t}\right\|_{L^{2}}^{2} d t+\alpha \int_{0}^{T} \mathcal{H}(t) \mathrm{d} t \\
& \quad+N_{2}(\psi)\left(T \mathcal{H}(0) \int_{0}^{T}\left\|\partial_{x} P_{e x t}\right\|_{L^{2}}^{2} \mathrm{~d} t\right)^{1 / 2}
\end{aligned}
$$


where

$$
\begin{aligned}
& C(m)=\|m\|_{L^{\infty}}+\frac{L}{2}\left\|\left(1-m_{x}\right)-1 / 2\right\|_{L^{\infty}}, \\
& N_{2}(\psi)=\sup _{t \in[0, T]} \frac{\left\|\Psi_{2}(t)\right\|_{L^{2}}}{\sqrt{\mathcal{H}(0)}} \quad \text { with } \Psi_{2}=\partial_{x}\left(\frac{1-m_{x}}{2} \psi+(x-m) \psi_{x}\right) .
\end{aligned}
$$

Proof. We split $\int_{0}^{L} P_{\text {ext }} \zeta \mathrm{d} x$ as the sum $A+B$ where

$$
A=\int_{0}^{L} P_{e x t} \partial_{x}(m \eta) \mathrm{d} x, \quad B=\int_{0}^{L} P_{e x t}\left(-\frac{1}{4} \eta+\frac{1-m_{x}}{2} \eta\right) \mathrm{d} x .
$$

Since $m(0)=m(L)=0$, one has $A=-\int_{0}^{L}\left(\partial_{x} P_{\text {ext }}\right) m \eta \mathrm{d} x$, and hence

$$
|A| \leq\left\|\partial_{x} P_{e x t}\right\|_{L^{2}}\|m\|_{L^{\infty}}\|\eta\|_{L^{2}} .
$$

On the other hand,

$$
|B| \leq \frac{1}{2}\left\|\left(1-m_{x}\right)-1 / 2\right\|_{L^{\infty}}\left\|P_{e x t}\right\|_{L^{2}}\|\eta\|_{L^{2}} .
$$

Now, since $P_{\text {ext }}$ has mean value zero by assumption (23), it follows from the Poincaré inequality that

$$
\left\|P_{\text {ext }}\right\|_{L^{2}} \leq L\left\|\partial_{x} P_{e x t}\right\|_{L^{2}} .
$$

By combining the previous inequalities, we conclude that

$$
\left|\int_{0}^{L} P_{e x t} \zeta \mathrm{d} x\right| \leq C(m)\left\|\partial_{x} P_{e x t}\right\|_{L^{2}}\|\eta\|_{L^{2}},
$$

which immediately implies that, for any $\alpha>0$,

$$
\begin{aligned}
\left|\int_{0}^{T} \int_{0}^{L} P_{e x t} \zeta \mathrm{d} x \mathrm{~d} t\right| & \leq \frac{1}{2 \alpha g} C(m)^{2} \int_{0}^{T}\left\|\partial_{x} P_{e x t}\right\|_{L^{2}}^{2} \mathrm{~d} t+\frac{\alpha g}{2} \int_{0}^{T}\|\eta\|_{L^{2}}^{2} \mathrm{~d} t \\
& \leq \frac{1}{2 \alpha g} C(m)^{2} \int_{0}^{T}\left\|\partial_{x} P_{e x t}\right\|_{L^{2}}^{2} \mathrm{~d} t+\alpha \int_{0}^{T} \mathcal{H}(t) \mathrm{d} t .
\end{aligned}
$$

It remains to estimate the terms which involve the Dirichlet to Neumann operator. In doing so, we use the following well-known formula (which follows from (32))

$$
G(\eta) \psi=-\partial_{x} \bar{V}
$$

Since $\bar{V}$ vanishes for $x=0$ or $x=L$, by integration by parts, we get

$$
\int_{0}^{L}\left(\frac{1-m_{x}}{2} \psi+(x-m) \psi_{x}\right) G(\eta) \psi \mathrm{d} x=\int_{0}^{L} \Psi_{2} \bar{V} \mathrm{~d} x .
$$

Since $\partial_{x} P_{e x t}=\chi \bar{V}$ by definition and since $\chi(x)=1$ on the support of $\Psi_{2}$ (by assumption on $m$ ), we deduce that

$$
\int_{0}^{L}\left(\frac{1-m_{x}}{2} \psi+(x-m) \psi_{x}\right) G(\eta) \psi \mathrm{d} x=\int_{0}^{L} \Psi_{2} \partial_{x} P_{e x t} \mathrm{~d} x .
$$

As a consequence,

$$
\begin{aligned}
\left|\int_{0}^{L}\left(\frac{1-m_{x}}{2} \psi+(x-m) \psi_{x}\right) G(\eta) \psi \mathrm{d} x\right| & \leq\left\|\Psi_{2}\right\|_{L^{2}}\left\|\partial_{x} P_{e x t}\right\|_{L^{2}} \\
& \leq N_{2, T}(\psi) \sqrt{\mathcal{H}(0)}\left\|\partial_{x} P_{e x t}\right\|_{L^{2}}
\end{aligned}
$$


by definition of $N_{2, T}(\psi)$. Then, using $\int_{0}^{T} f(t) \mathrm{d} t \leq \sqrt{T}\left(\int_{0}^{T} f(t)^{2} \mathrm{~d} t\right)^{1 / 2}$, we deduce that

$$
\begin{aligned}
& \mid \int_{0}^{T} \int_{0}^{L}\left(\frac{1-m_{x}}{2} \psi+(x-m) \psi_{x}\right) G(\eta) \psi \mathrm{d} x \mathrm{~d} t \mid \\
& \leq N_{2, T}(\psi)\left(T \mathcal{H}(0) \int_{0}^{T}\left\|\partial_{x} P_{\text {ext }}\right\|_{L^{2}}^{2} \mathrm{~d} t\right)^{1 / 2} .
\end{aligned}
$$

This completes the proof.

In view of the previous lemmas, it remains only to estimate the integrals

$$
\int_{0}^{T} \int_{0}^{L}\left(\partial_{x} P_{e x t}(t, x)\right)^{2} \mathrm{~d} x \mathrm{~d} t,\left.\quad \int \zeta \psi \mathrm{d} x\right|_{0} ^{T}
$$

Firstly, recall from (21) that

$$
\int_{0}^{T} \int_{0}^{L}\left(\partial_{x} P_{e x t}(t, x)\right)^{2} \mathrm{~d} x \mathrm{~d} t \leq \mathcal{H}(0)
$$

here we used the assumption $\chi \leq 1$. To estimate the second term, set

$$
B(t):=\int_{0}^{L} \zeta(t, x) \psi(t, x) \mathrm{d} x .
$$

So we have to estimate $B(T)-B(0)$. In fact, we will estimate the two terms separately. We begin by integrating by parts to write $B(t)$ under the form

$$
B(t)=\int_{0}^{L} \eta \Psi_{1} \mathrm{~d} x \quad \text { where } \quad \Psi_{1}:=-m \psi_{x}-\frac{1}{4} \psi+\frac{1-m_{x}}{2} \psi .
$$

As a result $B(t) \leq\|\eta\|_{L^{2}}\left\|\Psi_{1}\right\|_{L^{2}}$ and hence

$$
\left|\int_{0}^{L} \zeta \psi \mathrm{d} x\right|_{0}^{T} \mid \leq\|\eta(0)\|_{L^{2}}\left\|\Psi_{1}(0)\right\|_{L^{2}}+\|\eta(T)\|_{L^{2}}\left\|\Psi_{1}(T)\right\|_{L^{2}} .
$$

Remembering that

$$
N_{1, T}(\psi)=\sup _{t \in[0, T]} \frac{\left\|\Psi_{1}(t)\right\|_{L^{2}}}{\sqrt{\mathcal{H}(0)}}, \quad\|\eta(t)\|_{L^{2}} \leq \sqrt{\frac{2}{g}} \sqrt{\mathcal{H}(t)},
$$

and using again the fact that $\mathcal{H}$ is decreasing, we obtain the estimate

$$
\|\eta(0)\|_{L^{2}}\left\|\Psi_{1}(0)\right\|_{L^{2}}+\|\eta(T)\|_{L^{2}}\left\|\Psi_{1}(T)\right\|_{L^{2}} \leq \frac{2 \sqrt{2}}{\sqrt{g}} N_{1, T}(\psi) \mathcal{H}(0) .
$$

By combining the previous estimates, we end up with

$$
\left(\frac{1}{2}-c-\alpha\right) \int_{0}^{T} \mathcal{H}(t) \mathrm{d} t \leq\left\{\frac{C(m)^{2}}{2 \alpha g}+\sqrt{T} N_{2, T}(\psi)+\frac{2 \sqrt{2}}{\sqrt{g}} N_{1, T}(\psi)\right\} \mathcal{H}(0) .
$$

As explained at the beginning of this section, this completes the proof. 


\section{Appendix A. Uniform eStimates For the Linearized PRoblem}

In this appendix we consider Cauchy problem for the linearized water-wave equations. As already seen, one can reduce the analysis of the Cauchy problem to the case of periodic functions which are even in $x$. We thus assume in this section that $x$ belongs to the circle $S^{1}=\mathbb{R} /(2 \pi \mathbb{Z})$ and use Fourier analysis. Also, to simplify notations we assume that $g=1$ and that the fluid is infinitely deep (that is $h=+\infty$ ), so that $G(0)$ is the Fourier multiplier $\left|D_{x}\right|$ defined by $\left|D_{x}\right|\left(\sum \psi_{n} e^{i n x}\right)=\sum \psi_{n}|n| e^{i n x}$. The equations read

$$
\left\{\begin{array}{l}
\partial_{t} \eta=\left|D_{x}\right| \psi \\
\partial_{t} \psi+\eta+P_{e x t}=0
\end{array}\right.
$$

Set

$$
P_{\text {ext }}=-\partial_{x}^{-1}\left(\chi(x) \partial_{x}^{-1}\left|D_{x}\right| \psi\right),
$$

where $\chi \geq 0$ is a smooth compactly supported function, even in $x$, and where, by definition,

$$
\partial_{x}^{-1} \sum_{n \in \mathbb{Z}} \psi_{n} e^{i n x}=\sum_{n \in \mathbb{Z} \backslash\{0\}} \frac{\psi_{n}}{i n} e^{i n x} .
$$

For the linearized problem, this definition of $P_{\text {ext }}$ is equivalent to (20) (recall that we assume that $P_{\text {ext }}$ has mean value zero).

Proposition A.1 (Uniform estimates). Let $s \in[0,+\infty)$ be such that $2 s \in \mathbb{N}$. For any initial data $\left(\eta_{0}, \psi_{0}\right)$ in the Sobolev space $H^{s}\left(S^{1}\right) \times H^{s+\frac{1}{2}}\left(S^{1}\right)$, the Cauchy problem for (59)-(60) has a unique solution $(\eta, \psi) \in C^{0}\left([0,+\infty) ; H^{s}\left(S^{1}\right) \times H^{s+\frac{1}{2}}\left(S^{1}\right)\right)$. Moreover, there exists a constant $C_{s}$ depending only on such that, for any $t \geq 0$,

$$
\|\eta(t)\|_{H^{s}}+\|\psi(t)\|_{H^{s+\frac{1}{2}}} \leq C_{s}\|\eta(0)\|_{H^{s}}+C_{s}\|\psi(0)\|_{H^{s+\frac{1}{2}}} .
$$

Remark A.2. The quantities $N_{1, T}(\psi)$ and $N_{2, T}(\psi)$, as introduced in the statement of Theorem 2.3, are bounded by

$$
K_{1}(m) \frac{\|\psi(t)\|_{H^{1}}}{\mathcal{H}(0)}, \quad K_{2}(m) \frac{\|\psi(t)\|_{H^{2}}}{\mathcal{H}(0)} .
$$

The previous proposition implies that

$$
N_{1, T}(\psi) \lesssim \frac{\|(\eta(0), \psi(0))\|_{H^{\frac{1}{2}} \times H^{1}}}{\|(\eta(0), \psi(0))\|_{L^{2} \times \dot{H}^{\frac{1}{2}}}}, \quad N_{2, T}(\psi) \lesssim \frac{\|(\eta(0), \psi(0))\|_{H^{\frac{3}{2}} \times H^{2}}}{\|(\eta(0), \psi(0))\|_{L^{2} \times \dot{H}^{\frac{1}{2}}}} .
$$

As already mentioned, the ratios in the right-hand side measure the frequency localization of the initial data. This shows that, in this case, Theorem 2.3 gives a quantitative bound in terms of the frequency localization of the initial data.

Proof. The existence of a solution follows from classical arguments and we prove only the estimate (61). In doing so, it is convenient to symmetrize this system. Consider the Fourier multiplier $\left|D_{x}\right|^{\frac{1}{2}}$ and set $\theta=\left|D_{x}\right|^{\frac{1}{2}} \psi$, which means that, if $\psi=\sum_{n \in \mathbb{Z}} \psi_{n} e^{i n x}$, then $\theta=\sum_{n \in \mathbb{Z}} \sqrt{|n|} \psi_{n} e^{i n x}$. The equations can be written under the form

$$
\partial_{t} u+L u+P u=0
$$


where

$$
u=\left(\begin{array}{c}
\eta \\
\theta
\end{array}\right), \quad L=\left(\begin{array}{cc}
0 & -\left|D_{x}\right|^{\frac{1}{2}} \\
\left|D_{x}\right|^{\frac{1}{2}} & 0
\end{array}\right), \quad P=\left(\begin{array}{cc}
0 & 0 \\
0 & -\partial_{x}^{-1}\left|D_{x}\right|^{\frac{1}{2}}\left(\chi \partial_{x}^{-1}\left|D_{x}\right|^{\frac{1}{2}} \cdot\right)
\end{array}\right) .
$$

Denote by $(\cdot, \cdot)$ the scalar product in $L^{2}\left(S^{1}\right) \times L^{2}\left(S^{1}\right)$. We obtain $L^{2}$ estimates for $u$ by a simple integration by parts. Indeed, since $L=-L^{*}$, we obtain

$$
\frac{d}{d t}\|u\|_{L^{2}}^{2}+(P u, u)=0
$$

Now $(P u, u) \geq 0$, and hence we have the estimate $\|u(t)\|_{L^{2}} \leq\|u(0)\|_{L^{2}}$ for all $t \geq 0$.

To estimate the Sobolev norms of $u(t)$, we cannot simply commute spatial derivatives to the equation. Indeed, since $P$ is an operator with variable coefficients, the commutator between $P$ and spatial derivatives does not vanish and then using the Duhamel formula we would obtain a bound which is not uniform in $t$. To overcome this difficulty, we commute the time derivative $\partial_{t}$ with the equation. Set $\dot{u}=\partial_{t} u$. Then $\dot{u}$ solves the same equation, so the previous $L^{2}$-bound applied with $u$ replaced by $\dot{u}$ gives the estimate

$$
\left\|\partial_{t} u(t)\right\|_{L^{2}} \leq\left\|\partial_{t} u(0)\right\|_{L^{2}}
$$

On the other hand, using the equation (32) and the triangle inequality, we get

$$
\begin{aligned}
& \|L u(t)\|_{L^{2}} \leq\left\|\partial_{t} u(t)\right\|_{L^{2}}+\|P u(t)\|_{L^{2}}, \\
& \left\|\partial_{t} u(0)\right\|_{L^{2}} \leq\|L u(0)\|_{L^{2}}+\|P u(0)\|_{L^{2}} .
\end{aligned}
$$

By combining the previous estimates with the easy bounds

$$
\begin{aligned}
& \|u(t)\|_{H^{1 / 2}} \leq\|L u(t)\|_{L^{2}}+\|u(t)\|_{L^{2}}, \quad \quad\|L u(0)\|_{L^{2}} \leq\|u(0)\|_{H^{1 / 2}}, \\
& \|P u(t)\|_{L^{2}} \leq K\|u(t)\|_{L^{2}}, \quad\|P u(0)\|_{L^{2}} \leq K\|u(0)\|_{L^{2}},
\end{aligned}
$$

we conclude that

$$
\|u(t)\|_{H^{1 / 2}} \leq C\|u(0)\|_{H^{1 / 2}},
$$

for some constant $C$ independent of time. Iterating this argument, we obtain $\|u(t)\|_{H^{k / 2}} \leq C_{k}\|u(0)\|_{H^{k / 2}}$ for any integer $k$.

\section{Appendix B. Luke's VARIATIONAL PRINCIPLE}

Our goal in this section is to relate the function $\Theta$ with Luke's variational principle. Consider the case $P_{\text {ext }}=0$. Following Luke, the gravity water-wave system can be derived by minimizing the following Lagrangian:

$$
\mathcal{L}=\int_{t_{0}}^{t_{1}} \iint_{\Omega(t)} p \mathrm{~d} y \mathrm{~d} x \mathrm{~d} t=-\int_{t_{0}}^{t_{1}} \iint_{\Omega(t)}\left(\partial_{t} \phi+\frac{1}{2}\left|\nabla_{x, y} \phi\right|^{2}+g y\right) \mathrm{d} y \mathrm{~d} x \mathrm{~d} t .
$$

Now observe that

$$
\int_{-h}^{\eta} \partial_{t} \phi \mathrm{d} y=\partial_{t}\left(\int_{-h}^{\eta} \phi \mathrm{d} y\right)-\left(\partial_{t} \eta\right) \psi, \quad \iint_{\Omega} g y \mathrm{~d} y \mathrm{~d} x=\int_{0}^{L} \frac{g}{2} \eta^{2} \mathrm{~d} x-\frac{g L h^{2}}{2},
$$

and recall that $\partial_{t} \eta=G(\eta) \psi$ and also the fact that the kinetic energy is given by $\frac{1}{2} \int \psi G(\eta) \psi \mathrm{d} x$. We thus find that

$$
\mathcal{L}=\int_{t_{0}}^{t_{1}}(K(t)-P(t)) \mathrm{d} t+C
$$


where $C$ is a constant, depending only on $h, L, t_{0}, t_{1}$, which does not contribute to a variational principle). The previous identity relates $\mathcal{L}$ to the usual expression for the Lagrangian the difference between the averaged kinetic energy and the averaged potential energy.

Now, instead of (63), write

$$
\int_{-h}^{\eta} \partial_{t} \phi \mathrm{d} y=\partial_{t}\left(\int_{-h}^{\eta} \phi \mathrm{d} y+\psi \eta\right)-\eta \partial_{t} \psi,
$$

to obtain that the Lagrangian $\mathcal{L}$ can be written under the form

$$
\mathcal{L}=\mathcal{L}^{\prime}+C-\left.\int \eta \psi \mathrm{d} x\right|_{t=t_{0}} ^{t=t_{1}}
$$

where $C$ is as above and

$$
\mathcal{L}^{\prime}=\int_{t_{0}}^{t_{1}} \int\left(-\eta \partial_{t} \psi-\frac{g}{2} \eta^{2}-\frac{1}{2} \psi G(\eta) \psi\right) \mathrm{d} x \mathrm{~d} t .
$$

Now, by definition of $\Theta=-\eta \partial_{t} \psi-\frac{g}{2} \eta^{2}$, this gives

$$
\mathcal{L}^{\prime}=\int_{t_{0}}^{t_{1}} \int\left(\Theta-\frac{1}{2} \psi G(\eta) \psi\right) \mathrm{d} x \mathrm{~d} t .
$$

\section{Appendix C. Another integral identity}

In this section we prove an integral identity analogous to the one obtained in Theorem 2.1. The main difference between these two results is that they involve two different observation terms.

Theorem C.1. Let $m \in C^{\infty}([0, L])$ with $m(0)=m(L)=0$. Then, for any regular solution of (12) defined on the time interval $[0, T]$, there holds

$$
\begin{aligned}
\frac{1}{2} \int_{0}^{T} \mathcal{H}(t) \mathrm{d} t+\mathcal{P}= & -\int_{0}^{T} \int_{0}^{L} P_{\text {ext }}\left(\partial_{x}(m \eta)-\frac{1}{4} \eta\right) \mathrm{d} x \mathrm{~d} t \\
& +\frac{g}{2} \int_{0}^{T} \int_{0}^{L}\left(1-m_{x}\right) \eta^{2} \mathrm{~d} x \mathrm{~d} t \\
& +\frac{1}{2} \int_{0}^{T} \iint_{\Omega(t)}\left(1-m_{x}\right)\left(\phi_{x}^{2}-\phi_{y}^{2}\right) \mathrm{d} y \mathrm{~d} x \mathrm{~d} t \\
& -\left.\int_{0}^{L}\left(\partial_{x}(m \eta)-\frac{1}{4} \eta\right) \psi \mathrm{d} x\right|_{0} ^{T} \\
& +\int_{0}^{T} \int_{0}^{L}\left(\frac{3}{2} \eta_{x}-\frac{1}{2} \partial_{x}\left(m_{x} \eta\right)\right) \phi_{y} \phi_{x} \mathrm{~d} y \mathrm{~d} x \mathrm{~d} t
\end{aligned}
$$

where

$$
\mathcal{P}=\left.\int_{0}^{T} \int_{0}^{L}\left(\frac{1}{2} h+\frac{3-m_{x}}{4} \eta\right) \phi_{x}^{2}\right|_{y=-h} \mathrm{~d} x \mathrm{~d} t .
$$


Proof. We have already proved (see (47)) that

$$
\begin{aligned}
\frac{1}{2} \int \mathcal{H}(t) \mathrm{d} t= & -\iint P_{\text {ext }}\left(\partial_{x}(m \eta)-\frac{1}{4} \eta\right) \mathrm{d} x \mathrm{~d} t \\
& +\frac{g}{2} \iint\left(1-m_{x}\right) \eta^{2} \mathrm{~d} x \mathrm{~d} t \\
& -\left.\int\left(\partial_{x}(m \eta)-\frac{1}{4} \eta\right) \psi \mathrm{d} x\right|_{0} ^{T} \\
& -R_{a}-R_{c}+\frac{1}{2} R_{b},
\end{aligned}
$$

where $R_{a}$ is given by Proposition $5.1, R_{b}$ is given by Lemma 5.2 and $R_{c}$ is given by (46). Consequently, it remains only to prove that

$$
R_{a}+R_{b}-\frac{1}{2} R_{c}=\mathcal{P}+\mathcal{N}+\mathcal{B}
$$

where

$$
\begin{aligned}
\mathcal{P} & =\left.\iint\left(\frac{1}{2} h+\frac{3-m_{x}}{4} \eta\right) \phi_{x}^{2}\right|_{y=-h} \mathrm{~d} x \mathrm{~d} t \\
\mathcal{N} & =-\iiint\left(\frac{3}{2} \eta_{x}-\frac{1}{2} \partial_{x}\left(m_{x} \eta\right)\right) \phi_{y} \phi_{x} \mathrm{~d} y \mathrm{~d} x \mathrm{~d} t \\
\mathcal{B} & =\iiint \frac{m_{x}-1}{2}\left(\phi_{x}^{2}-\phi_{y}^{2}\right) \mathrm{d} y \mathrm{~d} x \mathrm{~d} t .
\end{aligned}
$$

Lemma C.2. Set

Then

$$
V=\left.\left(\partial_{x} \phi\right)\right|_{y=\eta}, \quad B=\left.\left(\partial_{y} \phi\right)\right|_{y=\eta} .
$$

$$
R_{a}=\frac{1}{2} \iint\left((G(\eta) \psi) m V+B m \psi_{x}\right) \mathrm{d} x \mathrm{~d} t
$$

Proof. Recall from (38) that

Now write

$$
R_{a}=\iint(G(\eta) \psi) m \psi_{x} \mathrm{~d} x \mathrm{~d} t+\iint(N(\eta) \psi) m \eta_{x} \mathrm{~d} x \mathrm{~d} t .
$$

$$
\begin{aligned}
(G(\eta) \psi) m & \psi_{x}+(N(\eta) \psi) m \eta_{x} \\
& =(G(\eta) \psi)\left(m \psi_{x}-m B \eta_{x}\right)+\left(\frac{1}{2} V^{2}+\frac{1}{2} B^{2}\right) m \eta_{x} \\
& =(G(\eta) \psi) m V+\left(\frac{1}{2} V^{2}+\frac{1}{2} B^{2}\right) m \eta_{x} \\
& =\frac{1}{2}(G(\eta) \psi) m V+\left[\left(\frac{1}{2} V^{2}+\frac{1}{2} B^{2}\right) m \eta_{x}+\frac{1}{2}(G(\eta) \psi) m V\right] \\
& =\frac{1}{2}(G(\eta) \psi) m V+\left[\left(\frac{1}{2} V^{2}+\frac{1}{2} B^{2}\right) m \eta_{x}+\frac{1}{2}\left(B-\eta_{x} V\right) m V\right] \\
& =\frac{1}{2}(G(\eta) \psi) m V+\left[\frac{1}{2} B^{2} m \eta_{x}+\frac{1}{2} B m V\right] \\
& =\frac{1}{2}(G(\eta) \psi) m V+\frac{1}{2} B m \psi_{x},
\end{aligned}
$$

which implies that $R_{a}$ can be written under the form (67). 
Next, we express $R_{a}, R_{b}$ and $R_{c}$ in terms of integrals of $\nabla_{x, y} \phi$.

Lemma C.3. There holds

$$
\begin{aligned}
R_{a}= & \iiint \frac{m_{x}}{2}\left(\left(\partial_{x} \phi\right)^{2}-\left(\partial_{y} \phi\right)^{2}\right) \mathrm{d} y \mathrm{~d} x \mathrm{~d} t \\
R_{b}= & \frac{1}{4} \iiint\left(\left(\partial_{x} \phi\right)^{2}-\left(\partial_{y} \phi\right)^{2}\right) \mathrm{d} y \mathrm{~d} x \mathrm{~d} t-\left.\frac{h}{4} \iint\left(\partial_{x} \phi\right)^{2}\right|_{y=-h} \mathrm{~d} x \mathrm{~d} t \\
R_{c}= & \frac{1}{2} \iiint\left[m_{x}\left(\left(\partial_{x} \phi\right)^{2}-\left(\partial_{y} \phi\right)^{2}\right)-2 m_{x x} y\left(\partial_{x} \phi\right)\left(\partial_{y} \phi\right)\right] \mathrm{d} y \mathrm{~d} x \mathrm{~d} t \\
& -\left.\frac{h}{2} \iint m_{x}\left(\partial_{x} \phi\right)^{2}\right|_{y=-h} \mathrm{~d} x \mathrm{~d} t
\end{aligned}
$$

Proof. To obtain these identities, we will write $R_{a}, R_{b}$ and $R_{c}$ under the form

$$
\iint u(t, x, \eta(t, x)) \mathrm{d} x \mathrm{~d} t+\iint f(t, x, \eta(t, x)) \eta_{x}(t, x) \mathrm{d} x \mathrm{~d} t
$$

and then apply the rule (53) whose statement is recalled here: for any functions $u=u(x, y)$ and $f=f(x, y)$ with $\left.f\right|_{x=0}=\left.f\right|_{x=L}=0$, one has

$$
\int u(x, \eta(x)) \mathrm{d} x+\int f(x, \eta) \eta_{x} \mathrm{~d} x=\iint\left(\partial_{y} u-\partial_{x} f\right) \mathrm{d} y \mathrm{~d} x+\int u(x,-h) \mathrm{d} x .
$$

Computation of $R_{a}$. Recall that

$$
R_{a}=\frac{1}{2} \iint\left[(G(\eta) \psi) m V+B m \psi_{x}\right] \mathrm{d} x \mathrm{~d} t
$$

By definition one has

$$
G(\eta) \psi=\left.\left(\partial_{y} \phi-\eta_{x} \partial_{x} \phi\right)\right|_{y=\eta}, \quad V=\left.\left(\partial_{x} \phi\right)\right|_{y=\eta}, \quad B=\left.\left(\partial_{y} \phi\right)\right|_{y=\eta},
$$

SO

$$
\frac{1}{2} \int\left[(G(\eta) \psi) m V+B m \psi_{x}\right] \mathrm{d} x=\int u(x, \eta) \mathrm{d} x+\int f(x, \eta) \eta_{x} \mathrm{~d} x
$$

with

$$
u(x, y)=m\left(\partial_{x} \phi\right)\left(\partial_{y} \phi\right), \quad f(x, y)=\frac{m}{2}\left(\left(\partial_{x} \phi\right)^{2}-\left(\partial_{y} \phi\right)^{2}\right) .
$$

Since $\left.f\right|_{x=0}=\left.f\right|_{x=L}=0$ and $\left.u\right|_{y=-h}=0$, it follows from (71) that

$$
\frac{1}{2} \int\left[(G(\eta) \psi) m V+B m \psi_{x}\right] \mathrm{d} x=\iint\left(\partial_{y} u-\partial_{x} f\right) \mathrm{d} y \mathrm{~d} x
$$

Now, using that $\phi$ solves $\partial_{x}^{2} \phi+\partial_{y}^{2} \phi=0$, we easily find that

$$
\partial_{y} u-\partial_{x} f=\frac{m_{x}}{2}\left(\left(\partial_{x} \phi\right)^{2}-\left(\partial_{y} \phi\right)^{2}\right),
$$

so we verify the identity (68) for $R_{a}$.

Computation of $R_{b}$. We have to compute

$$
\int u(x, \eta) \mathrm{d} x+\int f(x, \eta) \eta_{x} \mathrm{~d} x
$$

with

$$
u(x, y)=\frac{1}{4} y\left[\left(\partial_{x} \phi\right)^{2}-\left(\partial_{y} \phi\right)^{2}\right], \quad f(x, y)=\frac{1}{2} y\left(\partial_{x} \phi\right)\left(\partial_{y} \phi\right) .
$$

Since $m(0)=m(L)=0$, one has $\left.f\right|_{x=0}=\left.f\right|_{x=L}=0$ and hence the wanted identity for $R_{b}$ follows from (71). 
Computation of $R_{c}$. It remains only to compute

$$
R_{c}=\iint m_{x} \eta\left(\frac{1}{2} V^{2}-\frac{1}{2} B^{2}+B V \eta_{x}\right) \mathrm{d} x \mathrm{~d} t .
$$

As above we have

$$
\int m_{x} \eta\left(\frac{1}{2} V^{2}-\frac{1}{2} B^{2}+B V \eta_{x}\right) \mathrm{d} x=\int u(x, \eta) \mathrm{d} x+\int f(x, \eta) \eta_{x} \mathrm{~d} x
$$

with

$$
u(x, y)=\frac{1}{2} m_{x} y\left(\left(\partial_{x} \phi\right)^{2}-\left(\partial_{y} \phi\right)^{2}\right), \quad f(x, y)=m_{x} y\left(\partial_{x} \phi\right)\left(\partial_{y} \phi\right) .
$$

Since $\partial_{x} \phi$ vanishes for $x=L$, we have $\left.f\right|_{x=0}=\left.f\right|_{x=L}=0$. On the other hand, one has

$$
\begin{aligned}
& \left.u\right|_{y=-h}=\left.\frac{1}{2} m_{x}\left(\partial_{x} \phi\right)^{2}\right|_{y=-h}, \\
& \partial_{y} u-\partial_{x} f=\frac{1}{2} m_{x}\left[\left(\partial_{x} \phi\right)^{2}-\left(\partial_{y} \phi\right)^{2}\right]-m_{x x} y\left(\partial_{x} \phi\right)\left(\partial_{y} \phi\right),
\end{aligned}
$$

so (70) follows from (71).

Lemma C.4. There holds

$$
\begin{aligned}
& \iint \rho(x)\left(\phi_{x}^{2}-\phi_{y}^{2}\right) \mathrm{d} y \mathrm{~d} x-\left.h \int \rho(x) \phi_{x}^{2}\right|_{y=-h} \mathrm{~d} x \\
& \quad=\int \rho \eta \phi_{x}^{2}(x,-h) d x-2 \iint \rho \eta_{x} \phi_{y} \phi_{x} d y d x+2 \iint \rho_{x}(y-\eta) \phi_{y} \phi_{x} \mathrm{~d} y \mathrm{~d} x .
\end{aligned}
$$

Proof. Set, for some fixed $t$,

$$
u(x, y)=-\rho(x)(y-\eta(t, x))\left(\partial_{y} \phi\right)(t, x, y)^{2} .
$$

Then $u(x, \eta(t, x))=0$ and $u(x,-h)=0$ and hence $\int_{-h}^{\eta(t, x)} u_{y} d y=0$. On the other hand

$$
u_{y}=-2 \rho(y-\eta) \phi_{y} \phi_{y y}-\rho\left(\phi_{y}\right)^{2},
$$

so integrating on $y \in[-h, \eta(x)]$ and then on $x \in[0, L]$ we obtain, remembering that $\phi_{y y}=-\phi_{x x}$,

$$
0=\iint u_{y}=-\iint \rho \phi_{y}^{2}+2 \iint \rho(y-\eta)\left(\partial_{y} \phi\right)\left(\partial_{x}^{2} \phi\right) .
$$

Now set $v:=\rho(y-\eta)\left(\partial_{y} \phi\right)\left(\partial_{x} \phi\right)$ and write

$$
\begin{aligned}
& \iint \rho(y-\eta)\left(\partial_{y} \phi\right)\left(\partial_{x}^{2} \phi\right) \mathrm{d} y \mathrm{~d} x=\iint \partial_{x} v \mathrm{~d} y \mathrm{~d} x \\
& \quad=+\iint\left\{-\rho_{x}(y-\eta)\left(\partial_{y} \phi\right)\left(\partial_{x} \phi\right)+\rho \eta_{x}\left(\partial_{y} \phi\right)\left(\partial_{x} \phi\right)-\rho(y-\eta)\left(\partial_{y} \partial_{x} \phi\right)\left(\partial_{x} \phi\right)\right\} \mathrm{d} y \mathrm{~d} x .
\end{aligned}
$$

Observe that $\iint \partial_{x} v \mathrm{~d} y \mathrm{~d} x=0$ since $\left.\int v\right|_{x=0, L} d x=0$ and since $\left.v\right|_{y=\eta}=0$. We deduce that

$0=-\iint \rho \phi_{y}^{2}-2 \iint \rho(y-\eta)\left(\partial_{y} \partial_{x} \phi\right)\left(\partial_{x} \phi\right)+2 \iint \rho \eta_{x} \phi_{y} \phi_{x}-2 \iint \rho_{x}(y-\eta) \phi_{y} \phi_{x}$, 
SO

$$
\begin{aligned}
0= & -\iint \rho \phi_{y}^{2}-\iint \partial_{y}\left(\rho(y-\eta) \phi_{x}^{2}\right)+\iint \rho \phi_{x}^{2} \\
& +2 \iint \rho \eta_{x} \phi_{y} \phi_{x}-2 \iint \rho_{x}(y-\eta) \phi_{y} \phi_{x},
\end{aligned}
$$

and hence

$0=\iint \rho\left(\phi_{x}^{2}-\phi_{y}^{2}\right)-\int(h+\eta) \rho \phi_{x}^{2}(x,-h) d x+2 \iint \rho \eta_{x} \phi_{y} \phi_{x}-2 \iint \rho_{x}(y-\eta) \phi_{y} \phi_{x}$, which concludes the proof.

We are now in position to obtain (66) which will conclude the proof of the theorem. Firstly, we write

$$
R_{a}=\iiint \frac{1}{2}\left(\phi_{x}^{2}-\phi_{y}^{2}\right) \mathrm{d} y \mathrm{~d} x \mathrm{~d} t+\iiint \frac{m_{x}-1}{2}\left(\phi_{x}^{2}-\phi_{y}^{2}\right) \mathrm{d} y \mathrm{~d} x \mathrm{~d} t,
$$

to obtain that, using (72) with $\rho=1$,

$$
\begin{aligned}
R_{a}= & \left.\frac{1}{2} \iint(h+\eta) \phi_{x}^{2}\right|_{y=-h} \mathrm{~d} x \mathrm{~d} t \\
& -\iiint \eta_{x} \phi_{y} \phi_{x} \mathrm{~d} y \mathrm{~d} x \mathrm{~d} t+\iiint \frac{m_{x}-1}{2}\left(\phi_{x}^{2}-\phi_{y}^{2}\right) \mathrm{d} y \mathrm{~d} x \mathrm{~d} t .
\end{aligned}
$$

Directly from (72) applied with either $\rho=1$ or $\rho=m_{x}$, we find that

$$
R_{b}=\frac{1}{4} \iint \eta \phi_{x}^{2}(x,-h) \mathrm{d} x \mathrm{~d} t-\frac{1}{2} \iiint \eta_{x} \phi_{y} \phi_{x} \mathrm{~d} y \mathrm{~d} x \mathrm{~d} t,
$$

and

$$
\begin{aligned}
R_{c}= & -\iiint m_{x x} y \phi_{x} \phi_{y} \mathrm{~d} y \mathrm{~d} x \mathrm{~d} t \\
& +\frac{1}{2} \iint m_{x} \eta \phi_{x}^{2}(x,-h) \mathrm{d} x \mathrm{~d} t-\iiint m_{x} \eta_{x} \phi_{y} \phi_{x} \mathrm{~d} y \mathrm{~d} x \mathrm{~d} t \\
& +\iiint m_{x x}(y-\eta) \phi_{y} \phi_{x} \mathrm{~d} y \mathrm{~d} x \mathrm{~d} t
\end{aligned}
$$

which simplifies to

$$
R_{c}=+\frac{1}{2} \iint m_{x} \eta \phi_{x}^{2}(x,-h) \mathrm{d} x \mathrm{~d} t-\iiint \partial_{x}\left(m_{x} \eta\right) \phi_{y} \phi_{x} \mathrm{~d} y \mathrm{~d} x \mathrm{~d} t .
$$

We have proved (66) which concludes the proof of Theorem C.1.

\section{REFERENCES}

[1] Fatiha Alabau-Boussouira, Roger Brockett, Olivier Glass, Jérôme Le Rousseau, and Enrique Zuazua. Control of partial differential equations, volume 2048 of Lecture Notes in Mathematics. Springer, Heidelberg; Fondazione C.I.M.E., Florence, 2012.

[2] Thomas Alazard. Boundary observability of gravity water waves. arXiv:1506.08520, 2015.

[3] Thomas Alazard, Pietro Baldi, and Daniel Han-Kwan. Control of water waves. arXiv:1501.06366.

[4] Thomas Alazard, Nicolas Burq, and Claude Zuily. Cauchy theory for the gravity water waves system with non-localized initial data. Annales de l'Institut Henri Poincare (C) Non Linear Analysis, 33(2):337 - 395, 2016.

[5] Gregory R. Baker, Daniel I. Meiron, and Steven A. Orszag. Generalized vortex methods for free surface flow problems. ii: Radiating waves. Journal of Scientific Computing, 4(3):237-259, 1989. 
[6] T. Brooke Benjamin and Peter J. Olver. Hamiltonian structure, symmetries and conservation laws for water waves. J. Fluid Mech., 125:137-185, 1982.

[7] Umberto Biccari. Internal control for non-local Schrödinger and wave equations involving the fractional laplace operator. arXiv:1411.7800.

[8] Daniel J. Bodony. Analysis of sponge zones for computational fluid mechanics. J. Comput. Phys., 212(2):681 - 702, 2006.

[9] Félicien Bonnefoy. Experimental and numerical modelling of severe sea states. PhD thesis, Université de Nantes, March 2005.

[10] Joseph Boussinesq. Sur une importante simplification de la théorie des ondes que produisent, à la surface d'un liquide, l'emersion d'un solide ou l'impulsion d'un coup de vent. Ann. Sci. École Norm. Sup. (3), 27:9-42, 1910.

[11] Yusong Cao, Robert F Beck, and William W Schultz. An absorbing beach for numerical simulations of nonlinear waves in a wave tank. In Proc. 8th Intl. Workshop Water Waves and Floating Bodies, pages 17-20, 1993.

[12] Angel Castro, Diego Córdoba, Charles Fefferman, Francisco Gancedo, and Javier GómezSerrano. Finite time singularities for the free boundary incompressible Euler equations. Ann. of Math. (2), 178(3):1061-1134, 2013.

[13] Didier Clamond, Dorian Fructus, John Grue, and Øyvind Kristiansen. An efficient model for three-dimensional surface wave simulations. II. Generation and absorption. J. Comput. Phys., 205(2):686-705, 2005.

[14] Alain Clément. Coupling of two absorbing boundary conditions for $2 \mathrm{~d}$ time-domain simulations of free surface gravity waves. J. Comput. Phys., 126(1):139-151, 1996.

[15] Alain Clément. Benchmark test cases for Numerical Wave Absorption. Report on the 1st Workshop of ISOPE Numerical Wave Tank group;Montreal 1998. In 9th Int. Offshore and Polar Engineering Conf. ISOPE' 99, Brest, France, 1999.

[16] Walter Craig and Catherine Sulem. Numerical simulation of gravity waves. J. Comput. Phys., 108(1):73-83, 1993.

[17] Gaelle Duclos, Alain Clément, and Gontran Chatry. Absorption of outgoing waves in a numerical wave tank using a self-adaptive boundary condition. International Journal of Offshore and Polar Engineering, 11(03), 2001.

[18] Guillaume Ducrozet. Modelisation of nonlinear processes in generation and propagation of sea states with a spectral approach. PhD thesis, Université de Nantes ; Ecole Centrale de Nantes (ECN), November 2007.

[19] Stéphan T Grilli and Juan Horrillo. Numerical generation and absorption of fully nonlinear periodic waves. Journal of Engineering Mechanics, 123(10):1060-1069, 1997.

[20] Moshe Israeli and Steven A. Orszag. Approximation of radiation boundary conditions. J. Comput. Phys., 41(1):115 - 135, 1981.

[21] G. I. Jennings, D. Prigge, S. Carney, S. Karni, J. B. Rauch, and R. Abgrall. Water wave propagation in unbounded domains. part II: Numerical methods for fractional pdes. Journal of Computational Physics, 275:443-458, 102014.

[22] Geri I. Jennings, Smadar Karni, and Jeffrey Rauch. Water wave propagation in unbounded domains. Part I: nonreflecting boundaries. J. Comput. Phys., 276:729-739, 2014.

[23] Atle Jensen, Didier Clamond, Morten Huseby, and John Grue. On local and convective accelerations in steep wave events. Ocean engineering, 34(3):426-435, 2007.

[24] Vilmos Komornik. Exact controllability and stabilization. RAM: Research in Applied Mathematics. Masson, Paris; John Wiley \& Sons, Ltd., Chichester, 1994. The multiplier method.

[25] David Lannes. Water waves: mathematical analysis and asymptotics, volume 188 of Mathematical Surveys and Monographs. American Mathematical Society, Providence, RI, 2013.

[26] Jesper Larsen and Henry Dancy. Open boundaries in short wave simulations - a new approach. Coastal Engineering, 7(3):285-297, 1983.

[27] Bernard Le Méhauté. Progressive wave absorber. Journal of Hydraulic Research, 10(2):153-169, 1972.

[28] Jacques-Louis Lions. Exact controllability, stabilization and perturbations for distributed systems. SIAM Rev., 30(1):1-68, 1988. 
[29] Elaine Machtyngier. Exact controllability for the Schrödinger equation. SIAM J. Control Optim., 32(1):24-34, 1994.

[30] Elaine Machtyngier and Enrique Zuazua. Stabilization of the Schrödinger equation. Portugal. Math., 51(2):243-256, 1994.

[31] Benjamin Mélinand. A mathematical study of meteo and landslide tsunamis: the Proudman resonance. Nonlinearity, 28(11):4037-4080, 2015.

[32] Sorin Micu and Enrique Zuazua. An introduction to the controllability of partial differential equations. Quelques questions de théorie du contrôle. Sari, T., ed., Collection Travaux en Cours Hermann, 2005.

[33] Yvon Ouellet and I Datta. A survey of wave absorbers. Journal of hydraulic research, 24(4):265280, 1986.

[34] Russell M. Reid. Open loop control of water waves in an irregular domain. SIAM J. Control Optim., 24(4):789-796, 1986.

[35] Russell M. Reid. Control time for gravity-capillary waves on water. SIAM J. Control Optim., 33(5):1577-1586, 1995.

[36] Russell M. Reid and David L. Russell. Boundary control and stability of linear water waves. SIAM J. Control Optim., 23(1):111-121, 1985.

[37] Xavier Ros-Oton and Joaquim Serra. The Pohozaev identity for the fractional Laplacian. Arch. Ration. Mech. Anal., 213(2):587-628, 2014.

[38] Semyon V. Tsynkov. Numerical solution of problems on unbounded domains. A review. Appl. Numer. Math., 27(4):465-532, 1998. Absorbing boundary conditions.

[39] Marius Tucsnak and George Weiss. Observation and control for operator semigroups. Birkhäuser Advanced Texts: Basler Lehrbücher. [Birkhäuser Advanced Texts: Basel Textbooks]. Birkhäuser Verlag, Basel, 2009.

[40] Sijue Wu. Well-posedness in Sobolev spaces of the full water wave problem in 3-D. J. Amer. Math. Soc., 12(2):445-495, 1999.

[41] Vladimir E. Zakharov. Stability of periodic waves of finite amplitude on the surface of a deep fluid. Journal of Applied Mechanics and Technical Physics, 9(2):190-194, 1968.

Thomas Alazard

CNRS et Département de Mathématiques et Applications UMR 8553

École normale supérieure

45 rue d'Ulm

Paris F-75005, France 